\title{
Relational Observables in Gravity: a Review ${ }^{\star}$
}

\author{
Johannes TAMBORNINO
}

Laboratoire de Physique, ENS Lyon, CNRS-UMR 5672, 46 Allée d'Italie, Lyon 69007, France

E-mail: johannes.tambornino@ens-lyon.fr

Received August 31, 2011, in final form March 14, 2012; Published online March 28, 2012

http://dx.doi.org/10.3842/SIGMA.2012.017

\begin{abstract}
We present an overview on relational observables in gravity mainly from a loop quantum gravity perspective. The gauge group of general relativity is the diffeomorphism group of the underlying manifold. Consequently, general relativity is a totally constrained theory with vanishing canonical Hamiltonian. This fact, often referred to as the problem of time, provides the main conceptual difficulty towards the construction of gauge-invariant local observables. Nevertheless, within the framework of complete observables, that encode relations between dynamical fields, progress has been made during the last 20 years. Although analytic control over observables for full gravity is still lacking, perturbative calculations have been performed and within de-parameterizable toy models it was possible for the first time to construct a full set of gauge invariant observables for a background independent field theory. We review these developments and comment on their implications for quantum gravity.
\end{abstract}

Key words: Dirac observables; quantum gravity; problem of time; gauge invariance

2010 Mathematics Subject Classification: 83C45; 83C05; 81S05

\section{Introduction}

As already noted by Dirac [14] the observables of any first class constraint theory must be constant along the gauge orbits and therefore have vanishing Poisson brackets with all the constraints. In the case of general relativity the gauge group is the four-dimensional diffeomorphism group, therefore the theory is fully constrained meaning that the canonical Hamiltonian vanishes on the constraint surface $[1,15]$. Thus, gravitational observables do not evolve dynamically, which is sometimes referred to as the 'problem of time' [6, 40, 46].

This nonevolution of observable quantities in gravity is in conflict with our observations as we experience the gravitational interaction as a dynamical process. However, the resolution to this apparent paradox lies in the fact that the canonical Hamiltonian describes evolution in coordinate time which, due to general relativity's invariance under general coordinate transformations, is indeed meaningless. What we observe is evolution with respect to other fields, for example the matter content of the universe.

From a mathematical point of view it is easy to construct a specific class of observables, namely spacetime integrals over scalar densities. These quantities can easily be seen to commute with the four-dimensional diffeomorphism constraints. However, they do not possess a local interpretation, which renders them pretty much useless as far as local observations are concerned. Local observables are much harder to construct: consider for example a scalar field $\phi(\sigma)$. The action of the spatial diffeomorphism constraint on this field is given by $\partial_{a} \phi(\sigma)$. Unless $\phi(\sigma):=\phi_{0}$ is chosen constant it cannot be an observable, thus local fields do not qualify as observables for general relativity. In fact, as was noted by Torre [72], general relativistic observables have to include an infinite number of derivatives and are therefore very nonlocal.

\footnotetext{
*This paper is a contribution to the Special Issue "Loop Quantum Gravity and Cosmology". The full collection is available at http://www.emis.de/journals/SIGMA/LQGC.html
} 
These problems can partially be resolved in the framework of complete observables which, in its present form, was first introduced by Rovelli $[62,64]$. The main idea is to relate multiple gauge-dependent fields in a gauge-invariant way and thus a precise implementation of the idea that all that matters in general relativity are relations between dynamical quantities. The mathematical underpinning of this formalism was substantially enhanced by Dittrich in $[16,17]$ and presented in a form that makes it applicable for full general relativity.

However, applied to general relativity the formalism is rather complicated and therefore calculations could not be pushed beyond the formal level in full analyticity so far. How can local observables, such as for example gravitational waves, be described in a fully gauge-invariant way? Progress into that direction was made in $[18,19]$ where perturbation theory around a fixed spacetime was developed.

For full general relativity with realistic matter content the structure of the observable algebra is difficult to obtain analytically. However, it turns out that for certain matter models the theory de-parameterizes and a full set of gauge invariant observables can be derived. This was for example done using pressure-less dust fields [13, 29] or a scalar field [21, 65]. The matter fields used there play the role of a dynamically coupled physical reference frame. Disregarding their phenomenological viability these models allowed for a first time to analytically study the problem of observables in a fully background independent field theory. Thus, there is hope that the techniques used there can be generalized to realistic matter models such as ordinary standard model matter.

Whereas the question of observables for classical gravity is by now rather well understood, at least on a conceptual level, similar developments for quantum gravity are still lacking. There are candidate theories that provide a framework for quantum dynamics of the gravitational field at the Planck scale, among them loop quantum gravity [60,68], but the details of the physical Hilbert space (that is, the quantum analogue of the classical equivalence classes of solutions to Einstein's equations) remain to a large extent unexplored. Therefore, a systematic analysis of quantum gravitational observables could not be carried out so far.

However, there has been a lot of progress using finite dimensional toy models to understand the conceptual difficulties that arise when the principles of quantum mechanics are combined with background independence, most notably by Rovelli and collaborators (see for example [63], where a novel (relational) interpretation of quantum mechanics and the wave function is developed). Within these models strategies have been developed to encode dynamics in a timeless quantum system and it was shown how to re-obtain unitary evolution in some limit.

On the field theoretic side progress concerning observables for quantum gravity [21, 33] has mainly been made in the de-parameterizable models mentioned above, where the problem of time can be circumvented by choosing a dynamically coupled reference frame. However, as the material reference frame essentially remains classical all the way down to the Planck scale it is still under investigation in how far these models can be trusted in the deep quantum regime.

The field of observables for classical and quantum gravity is a field with a long history that still receives a lot of attention. Since the pioneering works of Dirac [15] and Arnowitt, Deser and Misner [1], decomposing spacetime into a one parameter family of spatial slices to cast the theory into Hamiltonian form, it became clear that the question of gauge-invariant (under the diffeomorphism group) quantities for gravity is strongly linked to the choice of a reference frame or observer and its physical description in terms of elementary fields. Bergmann and Komar $[6,7,8,44]$ were the first to systematically analyse this issue and constructed a set of spacetime scalars from gravitational degrees of freedom that serves as a dynamically coupled reference frame. Whereas the interplay between gauge-invariance and reference frames and the "problem of time' are rather well understood at the classical level, there remain many open questions concerning its implications for a quantum theory of gravity: does the concept of 'time' in its classical sense even make sense in the deep quantum regime? what is the role of an observer in 
a theory of quantum gravity? is there any viable probability interpretation? is time evolution unitary in the standard sense? See for example the reviews by Kuchař [45] or Isham [41] for a critical acclaim of interpretational problems that have to be overcome by any candidate theory of quantum gravity. Written almost 20 years ago most of the problems presented therein are still unsolved. See also the work of Barbour and collaborators [3, 4, 5] for a complementary relational view on the problem of time.

In this article we restrict ourselves mainly to the relational approach to the construction of observables for general relativity. We try to give an overview of the relevant developments but, due to the breadth of the field, such an overview is necessarily selective and represents the author's own view on the field.

\section{Complete observables for Hamiltonian constraint systems}

General relativity is a gauge theory with $\operatorname{Diff}(M)$ as a gauge group, that is, mathematically different solutions to Einstein's equations which are related by a diffeomorphism can not be distinguished from physical grounds. Such equivalence classes of solutions are the observables of the theory. In general relativity the gauge structure is strongly interlinked with the question of observers: different observers, related by a diffeomorphism, will use different solutions to Einstein's equations to describe the same physical situation. A strategy to compute observables that is especially well suited for this interlink is the method of complete observables, which we discuss in this section.

\subsection{A warmup example}

Before diving into the details of the mathematical formalism we discuss an almost trivial, but nevertheless illuminating, warmup example to illustrate the general idea. Consider a free nonrelativistic particle in one spatial dimension (hence, the configuration space is one-dimensional) with an action given by

$$
S[q]:=\int_{t_{1}}^{t_{2}} d t \frac{1}{2} m \dot{q}^{2}
$$

where $q$ is the position of the particle, $m$ its mass and a dot indicates a derivative with respect to $t$. Evaluating $S[q]$ on its extremal points gives the solutions $q(\tau)=q+\frac{p}{m}(\tau-t)$, where $p$ denotes the particle momentum. As $S[q]$ has no nontrivial symmetries, there are no constraints and therefore $q(\tau)$ is the time evolution of an observable. In nonrelativistic physics one usually assumes that the time parameter $t$ corresponds to some external clock which is not influenced by the dynamics of the system. However, this is not the case in general relativity due to background independence: there is no preferred notion of external time.

To mimic this feature of background independence one can reformulate the nonrelativistic particle and introduce an additional (unphysical) parameter $s$. Consider an action principle on an extended two-dimensional configuration space

$$
S_{\text {ext }}[q, t]:=\int_{s_{1}}^{s_{2}} d s \frac{1}{2} m \frac{q^{\prime 2}}{t^{\prime}},
$$

where a slash now denotes derivatives with respect to $s$. One can easily see that both actions generate the same equations of motion because (2.2) evaluated on paths $(q(s), t(s))$ gives the same value as (2.1) evaluated on paths $q(s(t))$. Obviously, $(2.2)$ is invariant under a general re-parameterization $s \rightarrow \tilde{s}:=f(s)$ where $f$ is an arbitrary smooth function. This is the analogue of the diffeomorphism symmetry in general relativity. Introducing momenta $p_{t}$ and $p$ 
associated to the extended configuration space variables $t$ and $q$ respectively one can perform a Legendre transformation to obtain a canonical formulation of that system. However, the Legendre transformation turns out to be singular, which leads to a constraint

$$
\mathfrak{c}:=p_{t}+\frac{p^{2}}{2 m}
$$

and the Hamiltonian of the extended system is given by

$$
H_{\mathrm{ext}}=N\left(q, p_{q}, t, p_{t}\right) \mathfrak{c}
$$

where $N\left(q, p_{q}, t, p_{t}\right)$ is an arbitrary function on the extended phase space, analogous to lapse and shift in the canonical formulation of general relativity.

All observables must have vanishing Poisson-brackets with the constraint and, as a consequence, do not evolve in parameter-time $s$. This is analogous to the 'problem of time' found in general relativity.

In this toy model there is an easy explanation and solution to this paradox: We introduced the additional parameter-time by hand, therefore evolution in $s$ is meaningless. From (2.1) we already know that the only observable is given by $q(\tau)$, that is, it encodes a relation between the two dynamical variables $q$ and $t$. In more detail the gauge orbits of $q$ and $t$, generated by the constraint $\mathfrak{c}$ are given by

$$
q(s):=\alpha_{\mathfrak{c}}^{s}(q)=q+\frac{p}{m} s, \quad t(s):=\alpha_{\mathfrak{c}}^{s}(t)=t+s .
$$

The latter can trivially be inverted for $s$, and for each fixed value $t(s) \stackrel{!}{=} \tau$ we can insert this into the first to obtain

$$
q(\tau)=q+\frac{p}{m}(\tau-t)
$$

which can directly be seen to commute with (2.3). As we will see soon this is an example of a more general construction principle for gauge-invariant observables. Considering the gauge orbits $\alpha_{\mathfrak{c}}^{s}(q)$ and $\alpha_{\mathfrak{c}}^{s}(t)$ of the two phase space functions $q$ and $t$ an observable is given by

$$
F_{q, t}^{\tau}:=\left.\alpha_{\mathfrak{c}}^{s}(q)\right|_{\alpha_{\mathfrak{c}}^{s}(t)=\tau},
$$

that is, the value of $q$ at that point of the gauge orbit where $t$ takes the value $\tau$. This is an example of the intuitive idea that in a background independent theory all that matters are relations between dynamical quantities. In the following we explain how this statement can be made mathematically precise.

\subsection{Partial or complete observables?}

Rovelli [60] has noted that gauge invariant observables à la (2.4) are not the only quantities of physical interest in general relativity: his argument is based on the observation that Einstein's equations are applied successfully to numerous astrophysical and cosmological measurements without even bothering about gauge-invariant observables. There one uses gauge-dependent partial observables (namely the metric, coordinate-distances, etc.) to describe what one observes. A natural resolution of this apparent paradox, from the relational point of view, is the following: the coordinates which one measures are the readings of some physical coordinate system (for example defined by the collection of all matter fields present in the universe). However, typically one does not bother to describe this collection of all matter fields in the universe as it is way to complicated. Thus, observable quantities seem gauge-dependent from the restricted point 
of view. When including equations for the dynamical evolution of all matter into this system one would expect that the partial observables of the restricted system emerge as the complete observables of the full system fixed to some gauge. This could possibly explain the fact that partial observables often seem to describe the correct phenomenology although they are not gauge invariant quantities.

This observation is realized in a precise way in the de-parameterizable matter models which we will discuss further down. Something similar is conjectured to happen when considering a universe filled with more realistic matter content. There one does not have enough analytic control on the expressions to actually prove that this is truly the case. But the (experimentally well established) fact that one can use Einstein's equations for gauge-dependent partial observables to describe physical observations is a strong indication into this direction.

Many of the results in loop quantum gravity, such as for example the calculation of the graviton propagator from a fully background independent point of view (see [67] for a review), are performed on the level of the kinematical Hilbert space, thus implicitly using gauge-dependent partial observables. From our point of view (apart from the fact that the gauge used to calculate the propagator is not explicitly known, and consequently also its physical interpretation) such calculations are perfectly fine as they are carried out in the semiclassical regime: by the same argument as for classical gravity one can conjecture the gauge-dependence of observable quantities to be related to our ignorance of the surrounding fields that provide a physical reference frame. Taking into account all these fields (which will not be possible practically) would render the quantum partial observables gauge invariant. At least in a regime where a sufficient number of degrees of freedom can still be treated classically (to provide the dynamical reference frame) this interpretation will always be possible. Whether a similar scheme holds in the deep quantum regime, where all degrees of freedom need to be treated quantum mechanically, is an open question, as one would encounter all the difficulties that occur within standard quantum mechanics when treating the measurement apparatus quantum mechanically as well. But for the moment we do not consider this too big a problem. Should there be any experimental contact with quantum gravity in the future this will first concern the semiclassical regime. However, it remains an interesting conceptual question.

\subsection{Detailed construction}

Consider the Hamiltonian formulation of a classical mechanical system with $d$ degrees of free$\operatorname{dom}^{1}$ : Let $\mathcal{M}$ be a $2 d$-dimensional symplectic manifold (the phase space) with symplectic 2 -form $\Omega \in \Lambda^{2}(\mathcal{M})$. We will be interested in the special case where $\mathcal{M}=T^{*} \mathcal{Q}$ has the structure of a cotangent bundle over some manifold $\mathcal{Q}$ (the configuration space) as this is the kind of structure that one gets when starting from an action principle and then performing a Legendre transformation. To each function $f \in C^{\infty}(\mathcal{M})$ one can associate a Hamiltonian vector field as

$$
\chi_{f}:=\Omega^{-1}(d f, \cdot),
$$

which in turn defines the Poisson bracket structure on $C^{\infty}(\mathcal{M})$ :

$$
\{\cdot, \cdot\}: \quad C^{\infty}(\mathcal{M}) \times C^{\infty}(\mathcal{M}) \rightarrow C^{\infty}(\mathcal{M}), \quad f, f^{\prime} \mapsto\left\{f, f^{\prime}\right\}:=\Omega\left(\chi_{f}, \chi_{f^{\prime}}\right) .
$$

Let $H \in C^{\infty}(\mathcal{M})$ be a function on phase space and $\chi_{H}$ the associated Hamiltonian vector field. Then one can define the flow of $f \in C^{\infty}(\mathcal{M})$ generated by $H$ as a one-parameter family of maps

$$
\alpha_{H}^{t}: \quad C^{\infty}(\mathcal{M}) \rightarrow C^{\infty}(\mathcal{M}), \quad f \mapsto \alpha_{H}^{t}(f),
$$

\footnotetext{
${ }^{1}$ For simplicity, assume here that $d$ is finite. With more care these methods easily generalize to the field theoretic case.
} 
where the flow $\alpha_{H}^{t}(y)$ of a point $y \in \mathcal{M}$ is the unique solution to the differential equation

$$
\frac{d}{d t} \alpha_{H}^{t}(y)=\chi_{H}\left(\alpha_{H}^{t}(y)\right), \quad \alpha_{H}^{0}(y)=y
$$

and the action on $C^{\infty}(\mathcal{M})$ is defined via pullback

$$
\alpha_{H}^{t}(f)(y):=f\left(\alpha_{H}^{t}(y)\right) \quad \forall f \in C^{\infty}(\mathcal{M}) .
$$

Among all possible phase space functions there is one of particular importance: the Hamiltonian $H \in C^{\infty}(\mathcal{M})$ which is related to the action functional $S$ of the Lagrangian formalism via a Legendre transformation. Dynamical evolution on phase space is defined via its flow: for any phase space function $f$ time evolution is given by

$$
f(t):=\alpha_{H}^{t}(f) .
$$

Now assume that there is a set of constraints ${ }^{2}\left\{\mathfrak{c}^{I}\right\}_{I \in \mathcal{I}}$ (for an index set $\mathcal{I}$ ) fulfilling the first class property, i.e. they form a closed Poisson algebra (possibly with structure functions) $\left\{\mathfrak{c}^{I}, \mathfrak{c}^{J}\right\}=$ $f_{K}^{I J} \mathfrak{c}^{K}$. Such first class constraints typically emerge when the action functional $S$ is invariant under gauge symmetries and, as a consequence, the Legendre transformation is degenerate. The constraint hypersurface $\mathcal{C}$ is defined to be the subspace $\mathcal{C} \subset \mathcal{M}$ where all the constraints vanish, i.e.

$$
\mathcal{C}:=\left\{y \in \mathcal{M} \mid \mathfrak{c}^{I}(y)=0 ; \forall I \in \mathcal{I}\right\},
$$

and any equality that holds at least on $\mathcal{C}$ is called a weak equality, denoted by $\simeq$ :

$$
f \simeq f^{\prime} \Leftrightarrow f(y)=f^{\prime}(y) \quad \forall y \in \mathcal{C}, \quad f, f^{\prime} \in C^{\infty}(\mathcal{M}) .
$$

Using (2.5) one can explicitly calculate the flow:

$$
\alpha_{H}^{t}(f)(y)=\sum_{n=0}^{\infty} \frac{t^{n}}{n !}\{H(y), f(y)\}_{(n)},
$$

where $\{H, f\}_{(0)}:=f$ and $\{H, f\}_{(n)}:=\left\{H,\{H, f\}_{(n-1)}\right\}$ iteratively. The flow fulfills the group property, i.e. $\alpha_{H}^{t}(f) \circ \alpha_{H}^{s}(f)=\alpha_{H}^{t+s}(f)$.

In this formalism a gauge transformation $\mathcal{G}$ is a map from the constraint surface to the constraint surface that can be written as a composition of flows generated by a first class set of constraints $\left\{\mathfrak{c}^{I}\right\}_{I \in \mathcal{I}}$,

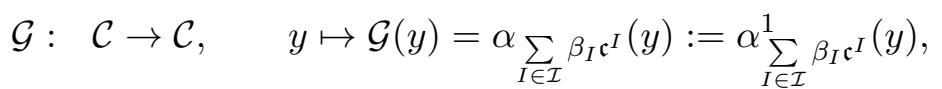

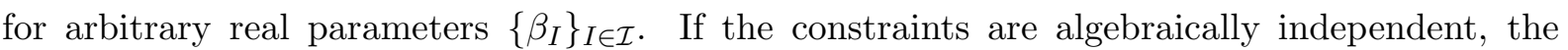
gauge transformations for each $y \in \mathcal{C}$ span an $|\mathcal{I}|$-dimensional submanifold of $\mathcal{C}$, the gauge orbit. Gauge transformations on $C^{\infty}(\mathcal{M})$ are then defined via pullback. Note that each gauge orbit remains unchanged if the first class set of constraints $\left\{\mathfrak{c}^{I}\right\}_{I \in \mathcal{I}}$ is replaced by an arbitrary linear combination thereof. That is, for an arbitrary nonsingular matrix $A_{J}^{I}(y)$, the gauge orbit defined by $\{\tilde{\mathfrak{c}}\}_{I \in \mathcal{I}}$ with $\tilde{\mathfrak{c}}^{I}=A_{J}^{I} \mathfrak{c}^{J}$ is exactly the same.

Physical observables must be gauge invariant, thus are constant along each gauge orbit. Therefore, gauge orbits and not points in phase space are the objects in phase space with an unambiguous physical interpretation. However, one can try to simplify this picture by fixing

\footnotetext{
${ }^{2}$ In field theories one will in general encounter an infinite number of constraints. In this case one has a set $\left\{\mathfrak{c}^{I}\right\}_{I \in \mathcal{I}}$ where $\mathcal{I}$ is an infinite index set.
} 
the gauge and picking out exactly one representative of each gauge orbit: this can be achieved by finding $|\mathcal{I}|$ algebraically independent phase space functions $T_{K}(y)$ and fix their values to $T_{K}(y) \stackrel{!}{=} \tau_{K}$. In order to ensure that the gauge fixing singles out one and only one point from each gauge orbit the following to conditions must be fulfilled (see e.g. [37]):

1. The chosen gauge must be accessible. That is, for each point $y \in \mathcal{C}$ there exists a gauge transformation $\mathcal{G}$ as defined in $(2.7)$ that maps the point $y$ onto a point $y^{\prime} \in \mathcal{C}$ fulfilling the gauge-fixing conditions $T_{K}\left(y^{\prime}\right) \simeq \tau_{K}$.

2. The gauge conditions $T_{K}(y) \simeq \tau_{K}$ must fix the gauge completely, i.e. there must be no other gauge transformation other than the identity left preserving $T_{K}(y) \simeq \tau_{K}$.

However, for an arbitrary gauge system there exists no constructive scheme that allows to find such a perfect set of gauge fixing conditions globally ${ }^{3}$ due to topological obstructions, so called Gribov ambiguities (see e.g. [71]).

An alternative approach, which circumvents the need to fix a specific gauge is the method of complete observables, based mainly on ideas of Rovelli ${ }^{4}$ (see $[62,64]$ and references therein). The starting point is that all observables are constants along each gauge orbit. Due to (2.6) this can be written as

$$
\left\{\mathfrak{c}^{I}, O\right\} \simeq 0 \quad \forall I \in \mathcal{I}
$$

These quantities are often called Dirac observables. For simple systems with one constraint Rovelli found a way to construct Dirac observables that have a very intuitive meaning: let $f, T \in C^{\infty}(\mathcal{M})$ be two arbitrary phase space functions and $\mathfrak{c} \in C^{\infty}(\mathcal{M})$ a single constraint. These phase space functions are referred to as partial observables, emphasizing the fact that these still contain gauge degrees of freedom. But one can combine these two in a relational manner to obtain a gauge invariant quantity,

$$
F_{f, T}^{\tau}(y):=\left.\alpha_{\mathfrak{c}}^{t}(f)(y)\right|_{\alpha_{\mathfrak{c}}^{t}(T)(y)=\tau} \cdot
$$

These gauge invariant quantities are then called complete observables. Their interpretation is the following: Let $f$ be a partial observable that associates a value to each point $y$ on the constraint surface $\mathcal{C}$. The constraint $\mathfrak{c}$ attaches a gauge orbit to each point $y$, given by $\alpha_{\mathfrak{c}}^{t}(y)$, parameterized by $t$. The same holds for $T(y)$. Now $F_{f, T}^{\tau}(y)$ associates to each point $y$ on the constraint surface the value $\alpha_{\mathfrak{c}}^{t_{0}}(f)(y)$ where the specific value $t_{0}$ for the parameter $t$ is given by $\alpha_{\mathfrak{c}}^{t_{0}}(T)(y)=\tau$. This construction ensures that the complete observables are constants along the gauge orbit and therefore commute with the constraints.

The properties of the above defined complete observable depend very much on the properties of the partial observables used in this construction. Practically one has to solve the relation $\alpha_{\mathfrak{c}}^{t}(T)(y)=\tau$ for $t$ in order to eliminate $t$ in (2.8). Generally there is no guaranty that the flow of $T$ can be uniquely solved for $t$ and the resulting complete observables (2.8) will generally be multi-valued. If one requires the complete observables to be unique one must demand that the phase space function $T$ has a monotonic behavior along the gauge orbit.

\footnotetext{
${ }^{3}$ As most investigations in gauge theories are carried out at a perturbative level, for many practical purposes this is not a severe problem: one fixes a gauge which fulfills the criteria stated above in a phase space region larger than the radius of convergence of the perturbation series. However, when interested in nonperturbative properties of a gauge theory, such as confinement of quarks in QCD or nonperturbative calculations in gravity, this is not possible. Therefore it is worth to develop alternative strategies to compute the physical degrees of freedom of a gauge theory. One of them, which fits very well with the interlink between gauge symmetry and background independence in general relativity is the method of complete observables.

${ }^{4} \mathrm{~A}$ different, however related, perspective was developed earlier by Bergmann and Komar, see for example [7, $8,44]$.
} 
The concept of complete observables generalizes to the case with more than one ${ }^{5}$ constraint following Dittrich [17]: for an arbitrary $|\mathcal{I}|$-dimensional first class constraint system $\left\{\mathfrak{c}^{I}\right\}_{I \in \mathcal{I}}$ a gauge transformation is given by (2.7). Analogous to (2.8), a complete observable can then be defined as a relation between a phase space function $f$ and a set of $|\mathcal{I}|$ algebraically independent phase space functions $T_{K}$, coined clock variables in this context:

$$
F_{f, T_{K}}^{\tau_{K}}(y)=\left.\alpha \sum_{I \in \mathcal{I}} \beta_{I^{\mathfrak{c}^{I}}}(f)(y)\right|_{\sum_{I \in \mathcal{I}} \beta_{I^{I}} \mathfrak{c}^{I}\left(T_{K}\right)(y)=\tau_{K}} .
$$

The interpretation is the the same as before, but the situation gets more complicated because the gauge orbit is not one-dimensional but $|\mathcal{I}|$-dimensional. In order to get a single-valued complete observable $F_{f, T_{K}}^{\tau_{K}}(y)$ all the equations $T_{K}(y)=\tau_{K}$ are required to have a unique solution ${ }^{6}$. One can rewrite (2.9) in a more convenient form realizing that the complete observables fulfill a set of differential equations:

$$
\frac{\partial}{\partial \tau_{L}} F_{f, T_{K}}^{\tau_{K}}(y) \simeq F_{\left(A^{-1}\right)_{M}^{L}\left\{\mathfrak{c}^{M}, f\right\}, T_{K}}^{\tau_{K}}(y), \quad F_{f, T_{K}}^{\tau_{K}=T_{K}(y)}(y)=f(y),
$$

where

$$
A_{K}^{J}:=\left\{\mathfrak{c}^{J}, T_{K}\right\}
$$

is an $|\mathcal{I}| \times|\mathcal{I}|$ matrix ${ }^{7}$ and $\left(A^{-1}\right)_{K}^{J} A_{J}^{L}=\delta_{K}^{L}$ and $A_{J}^{K}\left(A^{-1}\right)_{K}^{L}=\delta_{J}^{L}$ where summation over repeated indices is assumed. The (formal) solution to (2.10) in terms of an infinite sum is

$$
\begin{aligned}
F_{f, T_{K}}^{\tau_{K}} \simeq \sum_{n=0}^{\infty} & \frac{1}{n !}\left\{\tilde{\mathfrak{c}}^{K_{n}},\left\{\ldots,\left\{\tilde{\mathfrak{c}}^{K_{2}},\left\{\tilde{\mathfrak{c}}^{K_{1}}, f\right\}, \ldots\right\}\right.\right. \\
& \times\left(\tau_{K_{1}}-T_{K_{1}}\right)\left(\tau_{K_{2}}-T_{K_{2}}\right) \cdots\left(\tau_{K_{n}}-T_{K_{n}}\right),
\end{aligned}
$$

where $\tilde{\mathfrak{c}}^{K}:=\left(A^{-1}\right)_{J}^{K} \mathfrak{c}^{J}$.

By computing the Poisson bracket of (2.12) with the constraints one can check that it is indeed a weak Dirac observable. There one uses that the set of constraints $\left\{\tilde{\mathfrak{c}}^{I}\right\}_{I \in \mathcal{I}}$ is "weakly Abelian" (see below). Note that (2.12) so far is only a formal expression, neither there is a guarantee that $A_{K}^{J}$ can be inverted throughout the whole phase space nor that the infinite sum will eventually converge. These properties will in general depend strongly on the choice of the clocks $T_{K}$. However, (2.12) serves as the starting point for an illuminating perturbative analysis $[18,19]$, which we discuss in Section 3.3.

The constraints $\tilde{\mathfrak{c}}^{K}$ defined above describe the same gauge orbits as the original ones $\mathfrak{c}^{K}$ and furthermore fulfill the following important properties as can easily be calculated [17]:

$$
\left\{\tilde{\mathfrak{c}}^{J}, T_{K}\right\} \simeq \delta_{K}^{J}, \quad\left\{\tilde{\mathfrak{c}}^{K}, \tilde{\mathfrak{c}}^{L}\right\}=\mathcal{O}\left(\mathfrak{c}^{2}\right) \simeq 0
$$

where $\mathcal{O}\left(\mathfrak{c}^{2}\right)$ denotes terms at least quadratic in the constraints, for which reason the latter property is also referred to as 'weakly Abelian' in the literature. Introducing these weakly Abelian constraints instead of the original set was the key point that allowed Dittrich to express the Dirac observables of an arbitrary constraint system as an infinite power series as in (2.12).

\footnotetext{
${ }^{5}$ This construction includes the case of an infinite number of constraints, needed for the treatment of field theory. If $\mathcal{I}$ has uncountable cardinality the sum must be replaced by an appropriate integral in the following.

${ }^{6}$ Even if there is no unique solution to $T_{K}(y)=\tau_{K}$ it is possible to define the complete observable $F_{f, T_{K}}^{\tau_{K}}(y)$ if $\alpha \sum_{I \in \mathcal{I}} \beta_{I} \mathfrak{c}^{I}(f)$ takes the same value for all these solutions. This was formally achieved by introducing "partially invariant partial observables" (see [17]).

${ }^{7}$ In the case of an infinite dimensional constraint system $A_{K}^{J}\left(\sigma, \sigma^{\prime}\right)$ is an integral kernel where $K, J$ are now discrete indices and $\sigma, \sigma^{\prime}$ continuous ones.
} 
In Section 3.2.1 we will see that, for a peculiar class of clock fields, the above weak equalities can be replaced by strong equalities (i.e. they hold everywhere in phase space) which leads to further simplifications. For a detailed discussion of the mathematical properties of these complete observables from a symplectic geometric point of view see [69]. For example, one can show that associating complete observables to partial observables is an algebra morphism,

$$
F_{f \cdot g+h, T_{K}}^{\tau_{K}}=F_{f, T_{K}}^{\tau_{K}} \cdot F_{g, T_{K}}^{\tau_{K}}+F_{h, T_{K}}^{\tau_{K}} .
$$

\section{Complete observables for general relativity}

The formalism discussed in the last section can in principle be applied to any first class gauge system. However, the relational approach unfolds its true value when applied to general relativity. There the gauge symmetry (invariance under diffeomorphism of the four-dimensional manifold) is directly linked to the choice of observer which can ideally be accounted for within the relational approach. However, due to the complexity of the gauge group analytic results for full general relativity coupled to realistic matter fields have not been developed beyond the formal level so far. Nevertheless, there exist some interesting formal results mainly due to Dittrich [16]. Furthermore, perturbation theory has been developed which links the complete observables (2.12), evaluated in the neighborhood of a fixed phase space point of symmetry-reduced sector therein, to the dynamics of standard field theory on a given background $[18,19]$. Besides that, it turns out that the problem of calculating observables simplifies drastically when applying the formalism to general relativity coupled to a specific class of matter fields ${ }^{8}$. These matter fields are rather nonstandard and further research is needed to decide whether the specific models considered so far have any phenomenological significance. However, they provide examples where complete observables can be calculated exactly in a field theoretic context and therefore allow some insights in the gauge structure of general relativity. We discuss these developments in this section.

\subsection{General formalism}

The Hamiltonian form of general relativity ${ }^{9}$ was first introduced by Arnowitt, Deser and Misner in [1]. The 4-dimensional manifold $M=\mathbb{R} \times \Sigma$ is split into 3-dimensional spatial slices, the geometry on each slice $\Sigma_{t}$ is described by a Euclidean metric $q_{a b}(\sigma)$ and the canonical momentum $p^{a b}(\sigma)$ is related to the extrinsic curvature of these spatial slices as embedded in $M$. Thus, the phase space $\mathcal{M}$ of general relativity is given by the conjugate pair

$$
\left\{q_{a b}(\sigma), p^{c d}\left(\sigma^{\prime}\right)\right\}=\delta\left(\sigma, \sigma^{\prime}\right) \delta_{(a}^{c} \delta_{b)}^{d},
$$

where the round brackets denote symmetrization in the spatial indices. There are four constraints per spatial point, the (spatial) diffeomorphism constraint and the Hamiltonian (or scalar) constraint,

$$
\mathfrak{c}_{a}:=-\frac{2}{\kappa} q_{a c} D_{b} p^{b c}, \quad \mathfrak{c}:=\frac{1}{\kappa} \frac{1}{\sqrt{\operatorname{det} q}}\left[q_{a c} q_{b d}-\frac{1}{2} q_{a b} q_{c d}\right] p^{a b} p^{c d}-\sqrt{\operatorname{det} q} R(q),
$$

where $D_{b}$ denotes the covariant derivative, $R(q)$ the 3 -dimensional Ricci-scalar and $\kappa$ the gravitational coupling constant.

\footnotetext{
${ }^{8}$ This observation was first made by Brown and Kuchař in [13]. They are not explicitly using the language of relational observables there but the results are essentially the same.

${ }^{9}$ In what follows we mostly use the ADM-formulation of general relativity. At least as far as the classical theory is concerned the construction of observables follows the same scheme when using Ashtekar's variables. There the constraint algebra has to be supplemented by an additional SU(2)-Gauss constraint. However, as these constraints form an ideal in the full constraint algebra, this does not bring any additional obstructions.
} 
(Gauge-) dynamics in the ADM-formalism is encoded via the canonical Hamiltonian

$$
H_{\mathrm{can}}[N, \vec{N}]=\int d \sigma N(\sigma) \mathfrak{c}(\sigma)+N^{a}(\sigma) \mathfrak{c}_{a}(\sigma),
$$

which vanishes on the constraint hypersurface. The Lagrange multipliers $N$ and $N^{a}$ are known as lapse function and shift vector and encode the normal and tangential directions (to the spatial hypersurfaces $\Sigma_{t}$ ) of the dynamical flow. That the canonical Hamiltonian of general relativity is totally constrained is a feature shared with all background independent theories.

Defining the smeared constraints as

$$
\overrightarrow{\mathfrak{c}}(\vec{N}):=\int d \sigma N^{a}(\sigma) \mathfrak{c}_{a}(\sigma), \quad \mathfrak{c}(N):=\int d \sigma N(\sigma) \mathfrak{c}(s)
$$

the constraint algebra, sometimes called the Dirac or hypersurface deformation algebra, is given by

$$
\begin{aligned}
& \left\{\overrightarrow{\mathfrak{c}}(\vec{N}), \overrightarrow{\mathfrak{c}}\left(\overrightarrow{N^{\prime}}\right)\right\}=\kappa \overrightarrow{\mathfrak{c}}\left(\mathcal{L}_{\vec{N}} \overrightarrow{N^{\prime}}\right), \quad\{\overrightarrow{\mathfrak{c}}(\vec{N}), \mathfrak{c}(N)\}=\kappa \mathfrak{c}\left(\mathcal{L}_{\vec{N}} N\right), \\
& \left\{\mathfrak{c}(N), \mathfrak{c}\left(N^{\prime}\right)\right\}=\kappa \overrightarrow{\mathfrak{c}}\left(q^{-1}\left(N d N^{\prime}-N^{\prime} d N\right)\right),
\end{aligned}
$$

where $\mathcal{L}$ denotes the Lie-derivative. This is an infinite dimensional algebra, and furthermore the Poisson-bracket between two Hamiltonian constraints closes only with a (phase space dependent) structure function instead of a constant. Thus, the gauge structure of general relativity is very complicated compared to more common gauge theories such as $\mathrm{SU}(N)$-Yang-Mills theories. From a mathematical point of view this is the main reason why the problem of observables for general relativity is so hard.

However, in $[16,17]$ progress was made into that direction due to the following observation: in the canonical formulation of general relativity it is rather straightforward to construct quantities which are invariant under spatial diffeomorphism, e.g. by considering integrals of spatial scalars with density one over $\Sigma$. The construction of spacetime diffeomorphism invariant quantities, i.e. functions that additionally have vanishing Poisson brackets with the scalar constraint, is much harder, at least when one is interested in quantities that have a local spacetime interpretation. An interesting question is whether one can use these spatially diffeomorphism invariant quantities as a starting point to construct full observables for general relativity. The main obstruction which prevents a straightforward implementation of that idea is the fact that the diffeomorphism constraints do not form an ideal of the full Dirac algebra and the scalar constraints are no subalgebra. That is, starting from spatially diffeomorphism invariant quantities, the corresponding complete observables with respect to the scalar constraints will generally not be invariant under spatial diffeomorphisms.

However, in [17] it was shown that the complete observable (computed with respect to the full Dirac algebra (3.3)) $F_{f, T^{K}}^{\tau_{K}}$ for any spatially diffeomorphism invariant $f$ is (i) still weakly spatially diffeomorphism invariant and (ii) does not depend on the choice of clock variables associated to the spatial diffeomorphism constraints $\mathfrak{c}_{a}$, as long as all the clock variables commute with $\mathfrak{c}_{a}$. This further simplifies the construction of Dirac observables for general relativity as one can directly start with spatially diffeomorphism invariant quantities.

For a specific class of clock variables, namely functions which behave as spacetime scalars under the full spacetime diffeomorphism group, further simplifications occur. It turns out that the dynamical evolution of a spatial scalar $T(\sigma)$, which behaves under spatial diffeomorphisms as

$$
\left\{\mathfrak{c}_{a}(\sigma), T\left(\sigma^{\prime}\right)\right\}=\delta\left(\sigma, \sigma^{\prime}\right) \partial_{a} T(\sigma)
$$


gives rise to a spacetime scalar if and only if it has ultralocal ${ }^{10}$ Poisson brackets also with the Hamiltonian constraints

$$
\left\{\mathfrak{c}(\sigma), T\left(\sigma^{\prime}\right)\right\} \simeq \delta\left(\sigma, \sigma^{\prime}\right)
$$

Hence, when choosing spacetime scalars as clock variables the integral kernel (2.11) becomes ultralocal and the weakly Abelian constraints $\tilde{\mathfrak{c}}^{I}$ are local quantities, simply linear combinations of the original constraints. One can then show [16] that the complete observable $F_{\psi, T^{K}}^{\tau_{K}}$, where $\psi(\sigma)$ and the clock variables $T^{K}(\sigma), K=0,1,2,3$ are chosen as spacetime scalars, takes a particular simple form:

$$
\begin{aligned}
F_{\psi(\sigma), T^{K}}^{\tau_{K}} \simeq \sum_{n=0}^{\infty} \frac{1}{n !}\left\{\tilde{\mathfrak{c}}^{K_{n}}[1],\left\{\ldots,\left\{\tilde{\mathfrak{c}}^{K_{2}}[1],\left\{\tilde{\mathfrak{c}}^{K_{1}}[1], \psi(\sigma)\right\}, \ldots\right\}\right.\right. \\
\times\left(\tau_{K_{1}}-T_{K_{1}}\right)\left(\tau_{K_{2}}-T_{K_{2}}\right) \cdots\left(\tau_{K_{n}}-T_{K_{n}}\right)
\end{aligned}
$$

where

$$
\tilde{\mathfrak{c}}^{K}[1]:=\int d \sigma \tilde{\mathfrak{c}}^{K}(\sigma), \quad K=0,1,2,3
$$

is now a finite constraint system. That is, by an appropriate choice of clock variables the complete observables for general relativity can be seen to effectively depend only on 4 parameters $\tau^{K}$, $K=0,1,2,3$ instead of infinitely many. When all the spacetime scalars are additionally chosen invariant under spatial diffeomorphism the complete observables $F_{\psi, T^{0}}^{\tau}$ depend on one single parameter $\tau$ only, which then encodes the dynamical evolution of the observable as measured by a physical clock $T^{0}$.

One might wonder whether the choice of spacetime scalars as a starting point for complete observables for general relativity is to restrictive. However, it turns out that the clock-fields $T^{K}$, $K=0,1,2,3$ (chosen as spacetime scalars) can be used to transform any tensor on $M$ into a spacetime scalar, as long as $\operatorname{det} \partial_{\mu} T^{K} \neq 0$. For example, the inverse spacetime metric $g^{\mu \nu}$ can be lifted to a spacetime scalar as

$$
g^{K L}:=\left(\partial_{\mu} T^{K}\right)\left(\partial_{\nu} T^{L}\right) g^{\mu \nu} .
$$

The clock-fields $T^{K}$ can thus be understood as diffeomorphisms from the spacetime manifold $M$ to some 'physical' manifold $\mathcal{T}$ which is in turn defined through the possible values of the clock fields.

\subsection{Specific matter models as a dynamical reference frame}

The properties of the complete observables (2.12) or (3.4) depend crucially on the choice of clock variables $T^{K}$ which play the role of a dynamically coupled reference frame. First, the construction uses the weakly Abelian form of the constraint algebra (2.13), and in order to construct these constraints one has to invert a possibly infinite dimensional matrix. Second, so far there is no control over the radius of convergence (in phase space) of the infinite sum and the validity of (2.12) has to be checked case by case. In fact it is unlikely that one can find a set of clock variables which lets (2.12) converge throughout the whole phase space, as this would essentially be the same as finding a perfect gauge fixing for general relativity.

Thus, for general relativity with realistic matter content the observables are not under control analytically and one has to rely on perturbation theory (see further below). Besides that, it

\footnotetext{
${ }^{10} \mathrm{~A}$ symplectic structure is called ultralocal simply if it is proportional to the delta distribution.
} 
is interesting to study toy-systems where general relativity is coupled to very special ${ }^{11}$ matter models, where observables can be computed analytically. Such matter models include for example pressure-less dust-fields or scalar fields without potential. The common feature shared by all these matter models is that they fall into the class of de-parameterizable constraint systems. See for example [70] for a general discussion which matter models lead to de-parameterized constraint systems and also for an application of these ideas to phantom dust with negative energy density.

\subsubsection{De-parameterizable constraint systems}

A constraint system $\left\{\mathfrak{c}^{I}\right\}_{I \in \mathcal{I}}$ is said to be de-parameterizable if one can find a local coordinate chart on phase space with two mutually commuting sets of canonical pairs, denoted by $\left(q^{a}, p_{a}\right)$ and $\left(Q_{I}, P^{I}\right)$ such that in this chart the constraints can be written in the locally equivalent form $\mathfrak{c}^{I}=P^{I}+h^{I}(q, p)$ where $h^{I}$ depends only on $\left(q^{a}, p_{a}\right)$. Equivalence has to be understood in the sense that the constraint surface spanned by the two sets of constraints is the same. For such constraint systems the construction of observables simplifies a lot. Unfortunately general relativity does not fall into this class. However, by coupling the above mentioned matter models general relativity can be turned into a de-parameterizable theory. The hope therefore is that by analyzing these de-parameterizable toy models one can learn something about the observable structure for general relativity coupled to standard matter.

The reason why de-parameterizable constraint systems are particularly simple is the following: first class constraints of the form $\mathfrak{c}^{I}=P^{I}+h^{I}(q, p)$ necessarily form an Abelian constraint algebra due to linearity in the momenta $P^{I}$. The same holds for the generators $h^{I}$, they also form an Abelian algebra,

$$
\left\{h^{I}, h^{J}\right\}=0 .
$$

Furthermore, when choosing $Q^{I}$ as clock variables one realizes that property (2.13) now holds strongly, not only on the constraint surface:

$$
\left\{\mathfrak{c}^{I}, \mathfrak{c}^{J}\right\}=0, \quad\left\{\mathfrak{c}^{I}, Q_{J}\right\}=\delta_{J}^{I}
$$

For such systems there is no need to introduce weakly Abelian constraints, therefore one of the obstacles mentioned above, namely inverting the matrix $A_{I}^{J}$ is absent. When further restricting oneself to phase space functions $f(q, p)$ which do not depend on the clock variables $Q_{I}$ and their momenta $P^{I}$, the infinite sum (2.12) takes a much simpler form:

$$
F_{f(q, p), Q^{I}}^{\tau_{I}}=\left.\sum_{n=0}^{\infty} \frac{1}{n !}\left\{H_{\tau}, f\right\}_{(n)}\right|_{\tau^{K} \rightarrow\left(\tau^{K}-Q_{K}\right)},
$$

where the generator of the multi-fingered time evolution is then given by

$$
H_{\tau}:=\sum_{K} \tau^{K} h^{K}(q, p)
$$

Due to (3.6) and the first class property of the de-parameterized constraints $H_{\tau}$ is gauge invariant and further one finds

$$
\frac{\partial}{\partial \tau^{K}} F_{f(q, p), Q^{I}}^{\tau_{I}}=\left\{h^{K}, F_{f(q, p), Q^{I}}^{\tau_{I}}\right\}
$$

Thus, $H_{\tau}$ is the physical Hamiltonian that generates time evolution of observables in the dynamical reference frame defined by the clocks $Q^{I}$. Furthermore, because $H_{\tau}$ does not directly depend on the clock fields $Q^{I}$ this Hamiltonian is time-independent and generates conservative motion.

\footnotetext{
${ }^{11}$ We consider these matter models very special because they do not represent any of the known standard model matter fields. It is important to note that such matter models have been chosen for reasons of mathematical convenience and not because they provide any interesting phenomenology.
} 


\subsubsection{Dust as a dynamical reference system}

To give an explicit example of a matter-model that leads to de-parameterization for gravity in this section we will discuss pressure-less dust fields in some detail. We will mainly discuss the model used by Brown and Kuchař in [13] and later refined by Giesel, Hofmann, Thiemann and Winkler in [29, 30]. Similar models were also discussed for example by Kuchař and Torre [48], Rovelli [64] or Husain and Pawlowski [38, 39].

In [13] Brown and Kuchař realized that the combined system of pressure-less dust coupled to gravity can be brought into a de-parameterized form. They start from the dust action,

$$
S_{\text {dust }}=-\frac{1}{2} \int_{M} d^{4} X \sqrt{|\operatorname{det}(g)|} \rho\left[g^{\mu \nu} U_{\mu} U_{\nu}+1\right],
$$

which is coupled to the standard Einstein Hilbert action $S_{\mathrm{EH}}$. Here $U \in T^{*} M$ is a one-form defined as the differential $U=-d T+W_{j} d S^{j}, j=1,2,3$, for some scalar fields $T, W_{j}, S^{j}$. Thus, the dust action is a functional of the spacetime metric $g_{\mu \nu}$ and eight scalar fields $\rho, T, W_{i}, S^{i}$.

This action can be interpreted as a field theoretic generalization of the concept of free massive relativistic particles moving on geodesics of the gravitational field created by the entire collection of particles, see [13] for further details.

A canonical analysis (after solving some second class constraints that eliminate $\rho$ and $W_{j}$ and thereafter reducing the dimension of the phase space) leads to a first class system with constraints

$$
\mathfrak{c}^{\text {tot }}=\mathfrak{c}+\mathfrak{c}^{\text {dust }}, \quad \mathfrak{c}_{a}^{\text {tot }}=\mathfrak{c}_{a}+\mathfrak{c}_{a}^{\text {dust }},
$$

where the dust contributions are given by

$$
\mathfrak{c}^{\text {dust }}:=P \sqrt{1+\frac{1}{P^{2}} q_{a b}\left(\partial_{a} T+P_{j} \partial_{a} S^{j}\right)\left(\partial_{b} T+P_{j} \partial_{b} S^{j}\right)}, \quad \mathfrak{c}_{a}^{\text {dust }}:=P \partial_{a} T+P_{j} \partial_{a} S^{j},
$$

and the geometry contributions are as in (3.2). $P$ and $P_{j}$ are the momenta canonically conjugate to $T$ and $S^{j}$ respectively ${ }^{12}$. On the constraint hypersurface the constraints $\mathfrak{c}^{\text {tot }}$ and $\mathfrak{c}_{a}^{\text {tot }}$ can be solved for the momenta $P$ and $P_{j}$ respectively and be written in (partially) de-parameterized form as

$$
\begin{aligned}
& \tilde{\mathfrak{c}}^{\text {tot }}=P+h, \quad h=\sqrt{\mathfrak{c}^{2}-q^{a b} \mathfrak{c}_{a} \mathfrak{c}_{b}}, \\
& \tilde{\mathfrak{c}}_{j}^{\text {tot }}=P_{j}+h_{j}, \quad h_{j}=\left(S^{-1}\right)_{j}^{a}\left[\mathfrak{c}_{a}-h \partial_{a} T\right],
\end{aligned}
$$

when assuming that $\partial_{a} S^{j}$ is non degenerate and its inverse is given by $\left(S^{-1}\right)_{j}^{a}$. The total Hamiltonian constraint is in de-parameterized form now, but not the total spatial diffeomorphism constraint because $h_{j}$ still depends on $T$. Thus (3.7) and (3.8) form a strongly Abelian first class constraint algebra. Furthermore, $\left\{h(\sigma), h\left(\sigma^{\prime}\right)\right\}=0$ but in general $\left\{h(\sigma), h_{j}\left(\sigma^{\prime}\right)\right\} \neq 0$, $\left\{h_{i}(\sigma), h_{j}\left(\sigma^{\prime}\right)\right\} \neq 0$.

The fields $T$ and $S^{j}$ can then be used as clock-variables to compute a complete set of observables associated to the gravitational variables $q_{a b}$ and $p^{a b}$. In [13] the terminology of complete observables was not used yet, but it was already observed that one can use the clock variables $S^{j}$ to lift any spatial tensor on $\Sigma$ to the 'dust manifold' $\mathcal{S}:=S(\Sigma)$, as described in (3.5). That this is possible is essentially a consequence of the fact that $T$ and $S^{j}$ are spacetime scalars. This

\footnotetext{
${ }^{12}$ In [13] $P$ is chosen positive, which leads to a standard positive dust energy density. However, in [29] it was argued that the more natural choice from the point of view of relational observables is $P<0$. This means that the dust has negative energy density. However, the physical Hamiltonian that generates time evolution in the dust frame for the gravitational variables after a gauge reduction then turns out to be positive.
} 
can be interpreted as a gauge fixing $T(\sigma, t)=\tau, S^{j}(\sigma, t)=x^{j}$ where $\tau \in \mathbb{R}$ and $x \in \mathcal{S}$. Let $J(\sigma):=\operatorname{det}(\partial S / \partial \sigma)$ and consider

$$
\begin{aligned}
& \tilde{q}_{i j}(x)=\int_{\Sigma} d^{3} \sigma|J(\sigma)| \delta\left(x^{j}-S^{j}(\sigma)\right)\left(S^{-1}\right)_{i}^{a}\left(S^{-1}\right)_{j}^{b} q_{a b}(\sigma), \\
& \tilde{p}^{i j}(x)=\int_{\Sigma} d^{3} \sigma|J(\sigma)| \delta\left(x^{j}-S^{j}(\sigma)\right) \frac{\left(\partial_{a} S^{i}\right)\left(\partial_{b} S^{j}\right) p^{a b}}{J}(\sigma) .
\end{aligned}
$$

Remarkably it turns out that these two still form a canonical pair:

$$
\left\{\tilde{p}^{i j}(x), \tilde{q}_{k l}\left(x^{\prime}\right)\right\}=\kappa \delta_{(k}^{i} \delta_{l)}^{j} \delta\left(x, x^{\prime}\right) .
$$

The interpretation of (3.9) is obvious: These are the coordinate transformations of $\left(q_{a b}, p^{a b}\right)$ respectively into the dynamical coordinate system defined by $\sigma_{x}=S^{-1}(x)$.

In [29] Giesel, Hofmann, Thiemann and Winkler then re-derived and expanded these results using the terminology of complete observables: for each of the gravitational variables $f \in\left\{q_{a b}, p^{a b}\right\}$ one can calculate complete observables $F_{f,\left(T, S^{j}\right)}^{\left(\tau, x^{j}\right)}$ in the dynamical reference frame given by the readings $\left(\tau, x^{j}\right)$ of the dust-clocks $T, S^{j}$. These are given by

$$
\begin{aligned}
& Q_{j k}(\tau, x):=F_{q_{a b},\left(T, S^{j}\right)}^{\left(\tau, x^{j}\right)}:=\sum_{n=0}^{\infty} \frac{1}{n !}\left\{\tilde{h}_{\tau}, \tilde{q}_{j k}\right\}_{(n)}, \\
& P^{j k}(\tau, x):=F_{p^{a b},\left(T, S^{j}\right)}^{\left(\tau, x^{j}\right)}:=\sum_{n=0}^{\infty} \frac{1}{n !}\left\{\tilde{h}_{\tau}, \tilde{p}^{j k}\right\}_{(n)},
\end{aligned}
$$

where

$$
\tilde{h}_{\tau}:=\int_{\mathcal{S}} d^{3} x(\tau-\tilde{T}(x)) \tilde{h}(x) \quad \text { with } \quad \tilde{h}=h\left(\tilde{q}_{j k}, \tilde{p}^{j k}\right) .
$$

The expressions (3.10) can no longer be described in a compact form, they are rather complicated functions of $\tilde{q}, \tilde{p}$. However, from the reduced phase space point of view this is not a serious obstruction: the relevant quantities are the Dirac observables in the reduced phase space and the physical Hamiltonian which drives their time evolution with respect to the chosen clock fields. Setting $Q_{i j}(x):=Q_{i j}(\tau=0, x), P^{i j}(x):=P^{i j}(\tau=0, x)$, one can check through an explicit calculation that

$$
\left\{P^{i j}(x), Q_{k l}\left(x^{\prime}\right)\right\}=\kappa \delta_{(k}^{i} \delta_{l)}^{j} \delta\left(x, x^{\prime}\right)
$$

still form a canonical pair, all other Poisson brackets are vanishing. Then one can define

$$
H(\sigma)=\sqrt{C^{2}-Q^{i j} C_{i} C_{j}}(\sigma)
$$

where

$$
C:=\tilde{\mathfrak{c}}\left(\tilde{q}_{j k}=Q_{j k}, \tilde{p}^{j k}=P^{j k}\right), \quad C_{i}:=\tilde{\mathfrak{c}}_{i}\left(\tilde{q}_{j k}=Q_{j k}, \tilde{p}^{j k}=P^{j k}\right),
$$

and $\tilde{\mathfrak{c}}, \tilde{\mathfrak{c}}_{i}$ are the gravitational parts of the constraints, $\mathfrak{c}, \mathfrak{c}_{a}$, expressed in the dust coordinate system. The physical (i.e. nonvanishing) Hamiltonian in the dust frame is then given by

$$
H_{\text {dust }}:=\int_{\mathcal{S}} d^{3} x H(x)
$$


That is, for all observables $f\left(Q_{i j}, P^{i j}\right)$ evolution in the dust frame is given by

$$
\frac{d}{d \tau} f=\left\{H_{\text {dust }}, f\right\}
$$

The variation of the dust frame Hamiltonian is given by

$$
\begin{aligned}
\delta H_{\text {dust }} & =\int_{\mathcal{S}} d^{3} x\left[\left(\frac{C}{H}\right) \delta C-\left(\frac{Q^{i j} C_{j}}{H}\right) \delta C_{i}+\frac{1}{2 H} C_{i} C_{j} Q^{i k} Q^{j l} \delta Q_{i j}\right] \\
& =: \int_{\mathcal{S}} d^{3} x\left(N \delta C+N^{i} \delta C_{i}+\frac{1}{2} H N^{i} N^{j} \delta Q_{i j}\right) .
\end{aligned}
$$

Thus, the dust frame equations of motion generated by $H_{\text {dust }}$ are almost equivalent to the ones generated by the canonical Hamiltonian $H_{\text {can }}$ with the identification

$$
q_{a b}(\sigma) \rightarrow Q_{i j}(x), \quad p^{a b}(\sigma) \rightarrow P^{i j}(x)
$$

modulo the following important differences: first, lapse $N$ and shift $N^{i}$ are not phase space independent functions as for $H_{\text {can }}$ where they encode the arbitrariness of the foliation. Rather they are observable phase space functions composed out of the elementary fields $Q_{i j}, P^{i j}$ as

$$
N:=\frac{C}{H}, \quad N^{i}=-\frac{Q^{i j} C_{j}}{H} .
$$

Second, there is one additional contribution proportional to the Hamiltonian density $H(x)$.

The dust frame Hamiltonian has an infinite number of conserved charges, namely energy and momentum density $H(x), C_{j}(x)$. The latter ones generate active diffeomorphisms of the dust space $\mathcal{S}$, they are to be considered as symmetries of the system rather than gauge transformations generated by the passive diffeomorphisms of $\Sigma$. Likewise, the former are related in an intricate way to time re-parameterization invariance in general relativity.

From a mathematical point of view the analysis started in [13] and then re-derived in the language of complete observables in [29] is indeed very interesting: the observation that general relativity can be turned into a de-parameterizable theory when coupling to pressure-less dust fields allowed for a first time to analytically solve the problem of Dirac observables in a background independent field theory. This triggered further progress concerning the reduced phase space quantization approach to quantum gravity (see for example [21, 33, 39]) which we will comment on in Section 4. From a physical and phenomenological point of view the implications of that proposal are less clear: as the dust fields were introduced primarily for reasons of mathematical convenience it remains to be investigated whether the analysis can also be adapted to phenomenologically acceptable matter models such as standard model matter.

In [29, 30] Giesel, Hofmann, Thiemann and Winkler investigated in detail the phenomenological consequences of the dust model. Starting from the hypothesis that the dust fields are truly realized in nature they carefully worked out the consequences that distinguish this model from pure gravity plus standard matter in the cosmological sector. A priori there is one main obstacle to overcome: instead of the two degrees of freedom per spatial point of standard general relativity (two polarizations for a gravitational wave) the dust model has six degrees of freedom per spatial point. This is a consequence of the fact that passive diffeomorphism on $\Sigma$ are replaced by active diffeomorphism on $\mathcal{S}$, the four degrees of freedom present in the dust fields get re-absorbed by $Q_{a b}$ and $P^{a b}$, all their components are physically observable in the dust frame. However, dust energy- and momentum-density are conserved charges which essentially lets these extra degrees of freedom not dynamically propagate. This observation was used in $[29,30]$ to argue that the additional (i.e. not present in the standard treatment) terms found in first order cosmological perturbation theory can always be tuned arbitrarily small by an appropriate 
choice of conserved charges which brings this model into perfect agreement with cosmological observations. A similar conclusion was reached in [32] where the spherically symmetric sector of the dust model was analyzed. However, it is unlikely that the parameters of this model can be tuned in such a way to give negligible contributions to gravitational dynamics in any possible configuration of the gravitational field: as the dust fields do form singularities in finite time due to their self-gravitation (see for example [32] where such singularities where analyzed in the spherically symmetric sector) they will most likely not provide good clocks for extreme spacetime regions. This is the physical reflection of the mathematical problem to find a perfect gauge fixing which is valid everywhere in phase space. Furthermore, there is also the following conflict: in order to make the dust model compatible with phenomenology one would tune the dust energy- and momentum density as small as possible to have no measurable effect whatsoever. On the other hand, the clocks get worse the less energy they carry, heuristically speaking because they are influenced too much by the system to observe. Of course this argument holds not only for the dust model but needs to be clarified whenever additional fields are added to the setup just to serve as clock variables.

\subsubsection{Scalar fields as a dynamical reference system}

Besides the dust-models described above, scalar fields are also frequently used to provide a dynamically coupled reference frame. At least the Hamiltonian constraint can be brought into de-parameterized form when a single massless scalar field without potential is coupled to general relativity. This was first observed by Rovelli and Smolin in [65] and later studied in detail by Kuchař and Romano in [47].

The action for a massless scalar field without potential is given by

$$
S_{\phi}=\frac{1}{2} \int_{M} d^{4} X \sqrt{|\operatorname{det} g|} g^{\mu \nu}\left(\partial_{\mu} \phi\right)\left(\partial_{\nu} \phi\right)
$$

After a Legendre transform the canonical brackets are given by $\left\{\pi(\sigma), \phi\left(\sigma^{\prime}\right)\right\}=\delta\left(\sigma, \sigma^{\prime}\right)$, where $\pi$ is the scalar field momentum. A canonical analysis reveals that the scalar field contributions to diffeomorphism and Hamiltonian constraint are given by

$$
\mathfrak{c}_{a}^{\phi}=\pi \partial_{a} \phi, \quad \mathfrak{c}^{\phi}=\frac{1}{2 \sqrt{\operatorname{det} q}} \pi^{2}+\frac{1}{2} \sqrt{\operatorname{det} q} q^{a b}\left(\partial_{a} \phi\right)\left(\partial_{b} \phi\right)
$$

Because there is no potential term for the scalar field the Hamiltonian constraint for the coupled system gravity plus scalar field can be written in de-parameterized form as

$$
\tilde{\mathfrak{c}}^{\text {tot }}:=\pi-h, \quad h:=\sqrt{-\sqrt{\operatorname{det} q} \mathfrak{c}+\sqrt{\operatorname{det} q} \sqrt{\mathfrak{c}^{2}-q^{a b} \mathfrak{c}_{a} \mathfrak{c}_{b}} .}
$$

The constraints $\tilde{\mathfrak{c}}^{\text {tot }}, \mathfrak{c}_{a}^{\text {tot }}$ of the total system gravity plus scalar field span the same constraint surface as the original set $\mathfrak{c}^{\text {tot }}, \mathfrak{c}_{a}^{\text {tot }}$. Again, because $\tilde{\mathfrak{c}}^{\text {tot }}$ is of de-parameterized form these constraints form an Abelian algebra and furthermore $\{h, h\}=0$ strongly. Besides that, because $h$ does not depend on $\phi$ and $\pi$ it trivially commutes with the $\mathfrak{c}_{a}^{\phi}$. Therefore, the gauge-invariant (note that $h(\sigma)$ is of density weight one) physical Hamiltonian that generates time evolution with respect to the scalar field clock is given by

$$
H_{\phi}:=\int d \sigma h(\sigma)
$$

As described earlier, when considering already spatially diffeomorphism invariant partial observables $f$, the associated complete observables do not depend on the clock fields associated 
to the diffeomorphism constraints. Using $\phi$ as the physical clock associated to the Hamiltonian constraint to measure 'time' such complete observables are then given by (for spacetime scalars)

$$
F_{f, \phi}^{\tau}:=\sum_{n=0}^{\infty} \frac{\tau^{n}}{n !}\left\{H_{\phi}, f\right\}_{(n)},
$$

and therefore

$$
\frac{\partial}{\partial \tau} F_{f, \phi}^{\tau}=\left\{H_{\phi}, f\right\}
$$

For partial observables which are not yet invariant under spatial diffeomorphisms the dynamical evolution is more complicated. Therefore, this model is mainly applicable when the spatial diffeomorphism constraint has been solved already by other means. Recently Domagala, Giesel, Kaminski and Lewandowski [21] used this model as a starting point for a reduced phase space quantization where the diffeomorphism constraints are solved by standard methods on the kinematical Hilbert space of LQG and then dynamics with respect to this reduced Hamiltonian is implemented. This sheds some new light on the link between LQG and loop quantum cosmology where a scalar is also used commonly as a dynamical time variable, compare also [42, 43] for an analysis of related issues.

\subsection{Perturbative complete observables for general relativity}

For pure general relativity or general relativity coupled to standard matter the strategy described above does not work, essentially because it is not possible to rewrite the constraints such that they are linear in some momentum. For such generic configurations there is no scheme known so far that allows to compute gauge invariant observables analytically. However, in $[18,19]$ a scheme was proposed to compute complete observables approximately as perturbations around a fixed point in phase space (such as e.g. Minkowski space) or around a symmetry-reduced sector in phase space (e.g. the homogeneous-isotropic sector that describes cosmology). Within this scheme it can be seen (for an appropriate choice of clock variables) that the standard observables for field theory on a given background emerge as zeroth order terms of a perturbative expansion of the full background independent observables. Higher order terms include interactions with gravitational degrees of freedom, namely (classical) gravitons. Thus, the complete observables of full background independent gravity can be understood as a gauge invariant extension of observables of the linearized theory to higher orders.

Let us briefly describe the main strategy in the simpler case of perturbations around a fixed phase space point. The more general case of perturbations around a symmetry-reduced sector is discussed in [19].

The idea is essentially to expand the complete observable (2.12) around a fixed phase space point $Y_{0}$ : let $\mathcal{M}$ be a $2 d$-dimensional phase space with canonical coordinates $\left\{y^{a}\right\}_{a=1}^{2 d}$ and $\left\{\mathfrak{c}^{I}\right\}_{I \in \mathcal{I}}$ a first class constraint system with $|\mathcal{I}|$ algebraically independent constraints as in Section 2. Then choose a phase space point $Y_{0}$ on the constraint surface and define fluctuations around that phase space point as $\delta y^{a}:=y^{a}-Y_{0}^{a}$. Now simply expand all the constraints around $Y_{0}^{a}$,

$$
\mathfrak{c}^{I}={ }^{(0)} \mathfrak{c}^{I}\left(Y_{0}\right)+{ }^{(1)} \mathfrak{c}^{I}\left(Y_{0}, \delta y\right)+{ }^{(2)} \mathfrak{c}^{I}\left(Y_{0}, \delta y\right)+\cdots
$$

Here ${ }^{(0)} \mathfrak{c}^{I}$ depends only on the background variables $Y_{0}^{a},{ }^{(1)} \mathfrak{c}^{I}$ is linear in the fluctuations $\delta y^{a}$, ${ }^{(2)} \mathfrak{c}^{I}$ is quadratic in the fluctuations and so on. In general this expansion will have infinitely many terms if the constraints are nonpolynomial. Furthermore, the linearized constraints ${ }^{(1)} \mathfrak{c}^{I}$ fulfill an Abelian constraint algebra,

$$
\left\{{ }^{(1)} \mathfrak{c}^{I},{ }^{(1)} \mathfrak{c}^{J}\right\}=0 .
$$


Then, starting from (2.12) one can define an approximate complete observable ${ }^{[q]} F_{f, T^{K}}^{\tau_{K}}$ by simply restricting the full observable to terms that are at most of order $q$ in the fluctuations $\delta q$. These truncated complete observables do weakly commute with all the constraints $\mathfrak{c}^{I}$ up to terms of order $q$ in the fluctuation. In this sense ${ }^{[q]} F_{f_{i} T^{K}}^{\tau_{K}}$ define observables which are 'almost gauge invariant' in a precise manner and whose invariance properties get worse the farther one moves away from $Y_{0}$ in phase space. There is one technical obstruction though: the full complete observables (2.12) are defined using the weakly Abelian constraints (2.13). In order to compute these and to decide which terms are of order $q$ one has to invert the matrix $A_{I}^{K}=\left\{\mathfrak{c}_{I}, T^{J}\right\}$ for a given set of clock variables. In order to make $A_{I}^{J}$ perturbatively invertible the clocks have to be chosen such that the zeroth order of $A_{I}^{J}$ is invertible. Further simplifications occur when the clocks are chosen such that they are canonically conjugate to the linearized constraints. This is as far as general considerations can be carried out.

In [18] these ideas were applied to vacuum general relativity (in the Ashtekar formulation) and general relativity coupled to a massive scalar field perturbed around Minkowski space: pure vacuum general relativity has two physical degrees of freedom per spatial point. Therefore the ADM-parameterization of this phase space in terms of 3-metrics and 3-momenta as in (3.1) has a lot of redundant gauge degrees of freedom. In the linearized theory (linearized around Minkowski space) it is well known that the 'physical' (i.e. having vanishing Poisson brackets with the linearized constraints) are the traceless-transverse modes of the 3-metric (see for example the appendix of [18] for an explanation of this mode decomposition). Thus, one can conveniently use combinations of the 'unphysical' (from a linearized point of view) gravitational modes as clock variables. It turns out that in order to make the zeroth and first order of the complete observables agree with the observables of the linearized theory one can use a generalization of the so-called $A D M$ clocks $T_{\mathrm{ADM}}^{K}$ [1], certain rather nonlocal expressions involving all gravitational modes except the traceless-transverse ones which are canonically conjugate to the linearized constraints. Unlike scalar fields or dust fields discussed before these clock variables do not have a local interpretation, rather it turns out that they are related to translations at infinity for asymptotically flat spacetimes. Here we will not give the exact form of these clocks as functions of gravitational modes. Neither will we present the rather ugly computations that need to be performed to compute the approximate observables in that dynamical reference frame. All the details can be found in [18]. The main results are the following: for the case of a massive, minimally coupled scalar field of mass $m$ the total constraints are given by

$$
\mathfrak{c}_{a}^{\mathrm{tot}}:=\mathfrak{c}^{a}+\pi \partial_{a} \phi, \quad \mathfrak{c}^{\mathrm{tot}}:=\mathfrak{c}+\frac{1}{2}\left(\frac{1}{\sqrt{\operatorname{det} q}} \pi^{2} \sqrt{\operatorname{det} q} q^{a b} \partial_{a} \phi \partial_{b} \phi+\sqrt{\operatorname{det} q} m^{2} \phi^{2}\right),
$$

where the gravitational contributions are as in (3.2). Interested in the approximative complete observables ${ }^{[q]} F_{\phi, T_{\mathrm{ADM}}^{K}}^{\tau_{K}}$ associated to the scalar field in the dynamical reference frame defined by setting the $\mathrm{ADM}$ clocks to $T_{\mathrm{ADM}}^{0}=\tau, T_{\mathrm{ADM}}^{a}=0$ for low $q$ one finds the following.

- The zeroth order ${ }^{[0]} F_{\phi(\sigma), T_{\mathrm{ADM}}^{\tau}}^{\tau, 0}=0$ because the scalar field can be interpreted as a fluctuation around the fixed value $\Phi_{0}=0$.

- The first order ${ }^{[1]} F_{\phi(\sigma), T_{\mathrm{ADM}}^{\tau}}^{K}$ gives back the standard dynamical propagation of a free massive scalar field on a fixed Minkowski background:

$$
{ }^{[1]} F_{\phi(\sigma), T_{\mathrm{ADM}}^{K}}^{\tau, 0}=\int d \sigma^{\prime} \mathcal{S}_{\pi}\left(\tau, \sigma ; 0, \sigma^{\prime}\right) \pi\left(\sigma^{\prime}\right)+\mathcal{S}_{\phi}\left(\tau, \sigma ; 0, \sigma^{\prime}\right) \phi\left(\sigma^{\prime}\right),
$$

where the propagators are explicitly given by

$$
\mathcal{S}_{\pi}\left(\tau, \sigma ; 0, \sigma^{\prime}\right):=\cos \left[\sqrt{-\Delta_{\sigma}+m^{2}} \tau\right] \delta(\sigma, \sigma),
$$




$$
\mathcal{S}_{\phi}\left(\tau, \sigma ; 0, \sigma^{\prime}\right):=\frac{1}{\sqrt{-\Delta_{\sigma}+m^{2}}} \cos \left[\sqrt{-\Delta_{\sigma}+m^{2}} \tau\right] \delta(\sigma, \sigma)
$$

- The second order approximative complete observable ${ }^{[2]} F_{\phi(\sigma), T_{\mathrm{ADM}}^{\tau, 0}}^{K}$ was also computed explicitly in [18] and can schematically be written in a similar form

$$
{ }^{[2]} F_{\phi(\sigma), T_{\mathrm{ADM}}^{K}}^{\tau, 0}=\int d \sigma^{\prime} \mathcal{D}_{\pi}^{\text {grav }}\left(\tau, \sigma ; 0, \sigma^{\prime}\right) \pi\left(\sigma^{\prime}\right)+\mathcal{D}_{\phi}^{\text {grav }}\left(\tau, \sigma ; 0, \sigma^{\prime}\right) \phi\left(\sigma^{\prime}\right)+\text { gauge inv. ext. }
$$

where the propagators $\mathcal{D}_{\pi}^{\text {grav }}\left(\tau, \sigma ; 0, \sigma^{\prime}\right) \pi\left(\sigma^{\prime}\right)$ and $\mathcal{D}_{\phi}^{\text {grav }}\left(\tau, \sigma ; 0, \sigma^{\prime}\right)$ are modified such as to take interactions with the gravitational field into account and the gauge invariant extension term vanishes when all but the trace-free-transverse modes are set to zero. Thus, the second order complete observable can be interpreted as a free massive scalar field propagating on a (nonself-interacting) graviton background. Higher order corrections will also include interaction terms between the gravitational modes themselves.

The perturbative approach for complete observables was also used in [18] to analyze the 'spacetime algebra' of observables, that is their Poisson structure. Not surprisingly it was found that the Poisson brackets between two observables, evaluated at different physical times, depends drastically on the clocks used to define a dynamical reference frame. When computing the Poisson bracket between two scalar field observables to second order in the reference frame given by the ADM-clocks it turns out that the result is very similar to the flat spacetime case, namely

$$
\left\{{ }^{[2]} F_{\phi(\sigma), T_{\mathrm{ADM}}^{K}}^{\tau_{1}, 0}{ }^{[2]} F_{\phi(\sigma), T_{\mathrm{ADM}}^{K}}^{\tau_{2}=0,0}\right\} \propto-\mathcal{D}_{\pi}^{\text {grav }}\left(\tau_{1}, \sigma ; 0, \sigma^{\prime}\right)+\mathcal{O}(2),
$$

where $\mathcal{O}(2)$ refers to terms of second order. Compared to the flat spacetime case the propagator is replaced by an 'effective' propagator that takes into account to lowest order the effects of the nontrivial graviton background. When using scalar field clocks instead of the ADM-clocks as a dynamical reference frame one observes that (3.14) gets correction terms (additionally to the obvious 'translation' factor due to the use of a different set of clocks). Interestingly these correction terms are essentially of the form 'energy of the observed field divided by the energy of the clock fields'. Thus, in order to make these correction terms as small as possible one would increase the energy of the clock fields. This reflects the intuition that a physical clock is the better the more energy it has, simply because this way it is less influenced by the observed fields. However, this is in conflict with a different observation: as in general relativity any local energy density leads to local spacetime curvature one cannot use clock fields with arbitrarily high energies to describe almost Minkowskian spacetime configurations. Using the ADM-clocks which are rather nonlocal quantities this conflict is somehow circumvented. We will not go into the full field-theoretic description performed in [18] but illustrate this problem using a very simple toy example.

Consider two parameterized nonrelativistic, massless particles described by a constraint

$$
\mathfrak{c}:=p_{t}+\frac{p_{1}^{2}}{2 m_{1}}+\frac{p_{2}^{2}}{2 m_{2}}
$$

where $p_{t}$ is the momentum conjugate to the time variable $t$ and $p_{1}, p_{2}$ are the particle momenta conjugate to their respective position variables $q_{1}, q_{2}$. As already discussed in Section 2.1 a complete observable for this system is given by

$$
F_{q_{1}, t}^{\tau}=q_{1}+\frac{p_{1}}{m_{1}}(\tau-t) .
$$

This encodes the position $q_{1}$ of the first particle when the clock $t$ takes a value $\tau$. The Poisson bracket between two such observables at different times $\tau_{1}$ and $\tau_{2}$, analogous to the "spacetime 
algebra' in the field theoretic case, is given by

$$
\left\{F_{q_{1}, t}^{\tau_{1}}, F_{q_{1}, t}^{\tau_{2}}\right\}=\frac{1}{m_{1}}\left(\tau_{2}-\tau_{1}\right)
$$

Because this is a relational system without any preferred time variable one could also choose a different clock, namely $q_{2}$. The corresponding complete observable is then given by

$$
F_{q_{1}, t}^{\tilde{\tau}}=q_{1}+\frac{p_{1}}{m_{1}} \frac{m_{2}}{p_{2}}\left(\tilde{\tau}-q_{2}\right)
$$

and measures the position $q_{1}$ of the first particle when the clock $q_{2}$ takes a value $\tilde{\tau}$. Ignoring the interpretational difference between $\tau$ and $\tilde{\tau}$ this looks quite different. However, taking into account that the value $\tau$ is reached at that moment at which $\tilde{\tau}=F_{q_{2}, t}^{\tau}=q_{2}+\frac{p_{2}}{m_{2}}(\tau-t)$ and replaces $\tilde{\tau}$ in (3.17) one gets back (3.15). In so far, both complete observables describe the same evolution as long as the translation between different clocks is taken into account. However, this is not the case for the spacetime algebra:

$$
\left\{F_{q_{1}, q_{2}}^{\tilde{\tau}_{1}}, F_{q_{1}, q_{2}}^{\tilde{\tau}_{2}}\right\}=\left[\frac{1}{m_{1}}\left(\tilde{\tau}_{2}-\tilde{\tau}_{1}\right)\right]\left[\frac{m_{2}}{p_{2}}\right]\left[1+\frac{p_{1}^{2}}{2 m_{1}} \frac{2 m_{2}}{p_{2}^{2}}\right]
$$

The first factor is equal to the spacetime algebra of the complete observables with respect to the $t$-clock (3.16) and the second factor is due to the translation between $\tau \rightarrow \tilde{\tau}$. However, the third factor is nontrivial: there is a correction that occurs due to a nonstandard choice of clock that can be recognized as the energy of the observed particle divided by the energy of the clock. Intuitively this correction can be made arbitrary small by increasing the energy of the clock variable $q_{2}$. However, in a general relativistic setting this is problematic as discussed before. Thus, there seems to be a fundamental uncertainty when considering the spacetime algebra of observables already at the classical level. This might be related to the quantum uncertainties of relational observables derived by Giddings, Hartle and Marolf in [28].

The implications of this fundamental uncertainty for observables in full general relativity are still rather vague, as the computations performed in [18] are still on a preliminary level. However, the perturbative formalisms for complete observables might provide the right tools to further investigate such fundamental issues. In [19] this formalism was substantially expanded to accommodate for perturbations around a whole symmetry reduced sector in phase space, and as an application first order cosmological perturbation theory was re-derived from a fully background independent point of view.

An independent perturbative expansion for observables in classical and quantum gravity, in terms of inverse powers of a large cosmological constant, was studied by Gambini and Pullin in $[25,27]$.

\section{Observables for quantum general relativity}

As discussed in the previous section the situation concerning observables for classical general relativity is well understood, at least from a conceptual point of view. The method of complete observables implements the intuitive idea that observables in a background independent theory should encode relations between dynamical quantities. However, the situation of observables for quantum gravity is less clear which, to a large extent, is due to the fact that a generally agreed upon theory of quantum gravity does not exist yet. While loop gravity provides a kinematical framework for the quantum gravitational degrees of freedom and there exist well-motivated proposals for its dynamics, opinions differ in which exact way this framework is related to classical general relativity. Taking a conservative approach there are mainly two strategies concerning 
the problem of observables for quantum gravity: the Dirac approach or the reduced phase space approach. In the first one quantizes the whole phase space (resulting in the kinematical Hilbert space $\left.\mathcal{H}_{\text {kin }}\right)$ and then constructs quantum versions of the diffeomorphism and Hamiltonian constraint. The physical Hilbert space $\mathcal{H}_{\text {phys }}$, which contains states capturing the gauge invariant information of quantum gravity, is then essentially the joint kernel of all constraint operators. In the latter one first computes a complete set of gauge invariant observables of classical general relativity and subsequently only represent these on a Hilbert space. As one quantizes only gauge invariant degrees of freedom there is no need for any quantum constraint operators anymore, one directly constructs the physical Hilbert space. Besides these approaches, there exists also the point of view (as is argued for for example in [59]) that one should not strictly follow any 'quantization recipe' to construct quantum from classical gravity but rather propose the details of the theory by physical intuition and analogy with other quantum field theories and then check whether general relativity is contained in its semiclassical limit. Promisingly all these approaches seem to converge to roughly the same picture of quantum spacetime at the Planck scale [60, 68]. However, depending one one's point of view concerning the 'derivation' of quantum gravity the problem of quantum observables takes slightly different facettes. Within the reduced phase space approach the observable algebra is typically too complicated to be representable on some Hilbert space, therefore progress into this direction has been limited to the de-parameterizable models discussed above. Within the Dirac approach the physical Hilbert space is still under poor control due to its mathematical complexity which so far prevented progress concerning observables of the full theory. However, there are interesting toy models that allow to study the peculiarities of background independent quantum observables without diving into the details of full loop gravity. We will comment on both approaches below. Besides that, there has been progress to define quantum observables directly without constructing them from classical observables. In [52] Perez and Rovelli constructed so-called W-observables, directly defined on the space of diffeomorphism invariant spin network states ${ }^{13}$. These turn out to be related to n-point functions of group field theories. Whereas a clear connection to observables of classical gravity is absent, the same is true for n-point functions in for example quantum electrodynamics which have an inherent quantum interpretation.

An interesting, model independent analysis was presented by Giddings, Hartle and Marolf in [28]. Starting from classical diffeomorphism invariant, but nonlocal, expressions (spacetime integrals) they study in which limit these give rise to semilocal quantum operators once the theory is quantized. Interestingly they find that this is only possible for certain observables and certain states, related to the existence of an appropriate semiclassical limit of the theory. Furthermore, they derive bounds on a fundamental resolution for these observables due to the interplay of quantum-effects and general background independence. In model system this gives rise to a maximum number of degrees of freedom that can be contained in any finite volume.

\subsection{Dirac versus reduced phase space quantization}

Starting from a classical constraint system in general the two quantum theories obtained by Dirac quantization and reduced phase space quantization do not coincide. There are certain geometric systems with a high degree of symmetry where 'quantization commutes with reduction' [34], but in several simple model systems (see for example [49, 50, 53, 66]) it was found that the two quantum theories are substantially different, the spectra of physical operators do not coincide. From the point of view of complete observables this can be understood as follows:

\footnotetext{
${ }^{13}$ As usual in spinfoam models, the states of the theory are assumed to live on combinatorial rather embedded graphs. In this sense there is no nontrivial action of classical diffeomorphisms on these states. However, when passing from the quantum to the classical theory this action needs to be recovered, a point which is not clearly understood to date.
} 
following a reduced phase space quantization strategy the clock variables $T$ in the complete observables $F_{f, T}^{\tau}$ are replaced by real numbers (see [69] for details of this argument). Thus, the clocks $T$ are not quantized, their quantum fluctuations are suppressed. On the other hand, following the Dirac quantization algorithm also the clock variables $T$ are quantized, therefore the representations of complete observables within the Dirac approach will know about the quantum fluctuations of the clocks. In this sense, the Dirac quantization of a given constraint system can be regarded as more fundamental than the reduced phase space quantization of the same system, because all dynamical variables are treated on the same footing. In particular, when performing a reduced phase space quantization of complete observables with respect to different clock variables $T_{1} \neq T_{2}$ different quantum fluctuations will be suppressed and the two theories will in general be inequivalent. As already stated by Thiemann in [69]: "... the reduced phase space quantization of the Dirac observables can be useful only in a regime where the clocks $T_{j}$ can be assumed to behave classically. This is of course not the case with respect to any choice of clocks in extreme situations that we would like to access in quantum gravity such as at the big bang. There we necessarily need a constraint quantization of the system."

Subsequently, models for quantum gravity which rely on the technique of reduced phase space quantization need to be treated with care when their predictions for extreme spacetime regions are concerned because the clock variables are essentially treated as classical all the way down to the deep quantum regime. Nevertheless, such models can probably provide valuable insights into the quantum dynamics of the gravitational field in the semiclassical regime ${ }^{14}$.

For pure general relativity or general relativity with standard matter a reduced phase space quantization seems out of reach to date, simply because the Poisson structure on the reduced phase space is way to complicated to be faithfully represented on any known Hilbert space. Thus, a Dirac quantization remains the only option as far as the full theory is concerned. Efforts within the reduced phase space approach have so far been concentrated on de-parameterizable models obtained by coupling general relativity to some nonstandard matter as discussed in Section 3.2.

In the loop quantum gravity context the first such model was obtained by Rovelli and Smolin in [65] where they used the scalar field model discussed in Section 3.2.3. Using the scalar field $\phi$ as a clock they formally obtain the Schrödinger equation

$$
\left(i \hbar \frac{d}{d \phi}-\hat{H}_{\phi}\right) \psi=0
$$

where $\hat{H}_{\phi}$ is the quantum operator corresponding to the classical physical Hamiltonian in the scalar field reference system given by

$$
H_{\phi}=\int d \sigma \sqrt{-\sqrt{q} \mathfrak{c}}
$$

This operator is defined on the diffeomorphism invariant Hilbert space of (equivalence classes of) loops, therefore the contribution proportional to the diffeomorphism constraint in the classical expression (3.13) could be omitted.

Recently Domagala, Giesel, Kaminski and Lewandowski [21] built upon these ideas and showed explicitly that all steps of a reduced phase space quantization can be performed for this model using the rigorous mathematical tools from loop quantum gravity. The physical Hilbert space, including a physical inner product, can be constructed explicitly ${ }^{15}$ and the physical

\footnotetext{
${ }^{14}$ This might sound like a severe restriction on the fundamental validity of such models. However, from an observational point of view the restriction is less severe: if quantum gravity can be experimentally tested in the far future, this will be the regime of first interest.

${ }^{15} \mathrm{By}$ virtue of the reduced phase space quantization the physical Hilbert space for this model has exactly the same structure as the diffeomorphism invariant Hilbert space for loop quantum gravity. This is essentially possible because this model does not describe pure gravity but gravity coupled to a specific scalar field.
} 
Hamiltonian that generates time evolution in the classical reference frame defined by the scalar field $\phi$ can be constructed in analogy to the Hamiltonian constraint operator in loop quantum gravity.

Also the dust model discussed in Section 3.2.2 was used as the basis for a reduced phase space quantization. As the observable algebra (3.11) for the model system general relativity plus pressure-less dust takes exactly the same form as the kinematical algebra for general relativity in the ADM-formalism, the physical Hilbert space for this model is obtained immediately by standard techniques and coincides with the kinematical Hilbert space of loop quantum gravity in this case. Giesel and Thiemann [33] then used loop quantum gravity methods to construct an operator version of the dust-Hamiltonian (3.12) where the main obstacle is essentially to properly implement the square root. Thus, also for this system the reduced phase space program can be completed.

Due to the complicated structure of the physical Hamiltonians in both models as operators on the physical Hilbert space the analysis could not be pushed beyond the formal level so far. Interesting questions still to be answered in this framework concern the validity of the reduced phase space approach close to gravitational singularities, the observable effects of the dustor scalar field matter used to de-parameterize the system and the physical consequences of the chosen quantization of the Hamiltonian constraint. Recently progress into this direction has been made in the symmetry-reduced context, see [38] for an application of the dust model in cosmology. The fact that all these models posses a nonvanishing physical Hamiltonian as opposed to the vanishing Hamiltonian constraint in standard loop quantum gravity raises hope that scattering theory analogous to the S-matrix approach can be developed. The key ingredient, which is still missing and unfortunately very hard to achieve, are dynamically stable coherent states for the gravitational sector needed to implement the notion of 'asymptotically free states' in the scattering approach. See also [31] where the dust model was analyzed using an approximation of Born-Oppenheimer type for some preliminary steps into this direction.

\subsection{Relational quantum observables}

As discussed above the de-parameterizable models are very special because they can be written in a form where at least one of the momenta appears linear in the constraints and the remaining part does not depend on the associated position variable, schematically

$$
\mathfrak{c}=p_{t}+h(q, p) .
$$

The quantization of such models therefore can always be written in a Schrödinger-like form as in (4.1) and the associated time evolution can be implemented unitarily. Thus, due to the presence of a preferred reference frame (given by the dust fields or the scalar fields in the models discussed above) the usual incompatibility between general relativity and quantum mechanics, which manifests itself in the 'problem of time', is circumvented. However, the price to pay is that (i) there now is a fundamental preferred reference frame, and (ii) the dynamical clocks used to define space and time are essentially treated as classical variables. The first point is difficult to reconcile with the principles of general relativity and the second with the principles of quantum mechanics. Thus, beyond the semiclassical level one needs to be careful as far as a fully background independent theory of quantum gravity is concerned.

On the fundamental level the notion of a single universal time variable is meaningless and consequently there is no unitary time evolution. This is reflected in the Dirac quantization through the requirement that physical states be annihilated by the quantum constraint operators. However, at least in a semiclassical regime, unitary evolution of quantum states with respect to an emergent (possibly observer- and state-dependent) time variable must be possible. Because the physical Hilbert space of loop gravity is not well understood at the moment there is not much 
hope to clarify this issue, which is central to the interpretation of any quantum gravity theory, in the full theory. However, there has been a lot of effort to clarify the various problems that occur when one naively tries to reconcile quantum mechanics with the principle of background independence and thus constructs generally covariant quantum mechanics, most notably by Rovelli and collaborators [36, 51, 54, 55, 56, 58, 61, 63].

For example in [61] a simple two-dimensional model was analyzed with a classical constraint given by

$$
\mathfrak{c}=\frac{1}{1}\left(p_{1}^{2}+p_{2}^{2}+q_{1}^{2}+q_{2}^{2}\right)-M .
$$

It is clear that this model is not de-parameterizable and does not allow a global definition of time, therefore its quantization serves as a toy model for the above mentioned issues in quantum gravity. Nevertheless, one can define time variables locally using the framework of relational observables by considering for example $F_{q_{1}, q_{2}}^{\tau}$. The orbits in phase space defined by (4.2) are closed, therefore $F_{q_{1}, q_{2}}^{\tau}$ will only be a classically well defined observable for a finite range of physical time $\tau$ and furthermore multi-valued. This model can trivially be (Dirac-) quantized by starting from a kinematical Hilbert space $\mathcal{H}_{\text {kin }}:=L^{2}\left(\mathbb{R}^{2}\right)$ and then implementing the quantum constraint operator associated to (4.2) given by

$$
\hat{\mathfrak{c}}=-\hbar^{2} \frac{\partial^{2}}{\partial q_{1}^{2}}-\hbar^{2} \frac{\partial^{2}}{\partial q_{2}^{2}}+q_{1}^{2}+q_{2}^{2}-(2 j+1), \quad j \in \frac{\mathbb{N}}{2} .
$$

Note that the system has no quantum solutions unless for the values $M=2 j+1$. The physical Hilbert space $\mathcal{H}_{\text {phys }}$, defined as the kernel of $\hat{\mathfrak{c}}$, including a well defined inner product, can then explicitly be constructed.

A priori this physical Hilbert space comes without any notion of time or unitary evolution of states: the quantum observables $\hat{F}_{q_{1}, q_{2}}^{\tau}$ can be implemented as well defined self-adjoint operators on $\mathcal{H}_{\text {phys }}$ but generally do not encode unitary evolution of $q_{1}$ with respect to $q_{2}$. However, it turns out that for an appropriate choice of interval $\left[\tau_{1}, \tau_{2}\right]$ and quantum state $\psi\left(q_{1}, q_{2}\right) \hat{F}_{q_{1}, q_{2}}^{\tau}$ is the precise analog of the Heisenberg operator $\hat{q}(t)=e^{i \hat{H} t} \hat{q} e^{-i \hat{H} t}$ and describes almost unitary evolution of $q_{2}$ with respect to $q_{1}$. Eventually the restrictions on state and time interval do not hold anymore and unitary evolution breaks down.

An interesting development concerning unitary dynamics in timeless systems was recently presented by Bojowald, Höhn and Tsobanjan [10, 11]: they use the effective approach towards quantum constraint systems (see for example [12]) where the Hilbert space picture is essentially replaced by classical equations for expectation values and moments of the quantum operators. Generally one has to consider an infinite number of such momenta but for an appropriate choice of states higher order momenta are proportional to higher powers of $\hbar$ and can therefore be neglected for semiclassical considerations at a given precision. Their approach differs from the operator approach discussed above in so far as the observables, coined 'fashionables' in that context, encode correlations between expectation values. Thus, they are explicitly state dependent and valid only locally. However, in contrast to other approaches a transition between different clocks in the quantum theory is always possible. The authors show that although a definition of unitary evolution for arbitrary long timescales is not possible in timeless systems one can choose different time variables for different regimes and then patch together these intervals of unitary evolution in a consistent way, at least as long as semiclassical behavior for at least some dynamical quantities can be assumed. For a highly excited quantum system (such as quantum gravity close to the big bang) this description breaks down and nicely illustrates the intuition that quantum mechanical time evolution cannot be more than a semiclassical concept.

Recently Gambini, Pullin and collaborators have argued that the fundamental interpretation of quantum mechanics needs to be changed in order to accommodate for background independent quantum gravity where a strict separation of nondynamical measurement devices from 
the quantum dynamics of the system is not possible. See for example [22, 23, 24, 26] for the framework coined the 'Montevideo interpretation' of quantum mechanics.

\subsection{Spectra of physical operators in loop quantum gravity?}

One of the most interesting features of loop quantum gravity is that geometrical operators, such as the quantum versions of areas and volumina, have discrete spectra. Thus, the intuitive idea that smooth spacetime is replaced by a fundamentally discrete entity at the Planck scale is realized in a precise way in that formalism. Moreover, this Planck scale discreteness is not put in by hand but emerges naturally in the background independent quantization used in loop quantum gravity.

One of the open questions, still under debate, is whether the discreteness of these spectra is merely a kinematical artefact or also holds on the level of the physical Hilbert space. Area- and volume-operators are defined on the kinematical (or, depending on the interpretation, spatial diffeomorphism invariant) Hilbert space, and a priori one would have to construct their gaugeinvariant extensions to construct geometrical operators acting on the physical Hilbert space. However, this Hilbert space is under poor control so far. Thus, a detailed analysis as for the toy model presented above is not available.

In [20] Dittrich and Thiemann analyzed several finite dimensional models and showed that a priori an operator that has discrete spectrum on the kinematical Hilbert space may have continuous spectrum when extended in a gauge invariant way to the physical Hilbert space. Thus, they conclude that a more detailed analysis within the context of full loop quantum gravity is needed to decide whether physical areas and volumina are discrete or not.

In [57] Rovelli argued that the models considered in [20] show some pathological behavior not present in full loop quantum gravity related to a subtlety of compact versus noncompact orbits of the corresponding classical observables in phase space. Furthermore, a number of general physical arguments is provided in favor of the hypothesis that geometrical spectra remain discrete on the physical Hilbert space. One argument, which fits within the recent attempts to construct a reduced phase space quantization of gravity discussed above is the following: in general relativity the evolution of coordinate-dependent quantities is under-determined. This can be interpreted in two different ways. Either, one takes the point of view that only coordinateindependent quantities can possibly have a physical meaning. This is essentially the point of view taken when constructing complete observables for pure general relativity or general relativity with standard matter and subsequently applying a Dirac quantization. Or, one takes the point of view that the coordinates $x^{\mu}$ describe physical positions with respect to some physical dynamical reference frame which, however, one does not care describing. The underdeterminacy of the evolution then reflects the fact that the dynamical evolution of the reference frame is not taken into account ${ }^{16}$. Thus the seemingly gauge-dependent quantities are truly gauge-invariant observables of the extended system where the clock fields have been gauge fixed to some physical value.

This idea is realized in a precise way in the reduced phase space quantizations for gravity [21, $33,39]$ where discrete spectra for geometrical operators on the physical Hilbert space are found. However, as expressed above there are certain doubts concerning the validity of the reduced phase space quantization in the deep quantum regime and, consequently, whether physical predictions of these models at the Planck scale must be corrected or not.

Furthermore, one can argue that the measurable discreteness of the spectrum of some operator might be a kinematical property and not a dynamical one after all: consider a classical system where the motion of a free particle is constrained to a circle, thus its position is $q \in[0,2 \pi]$.

\footnotetext{
${ }^{16}$ See also [35] where the construction of complete observables for extended systems is studied and a similar conclusion is reached.
} 
Upon quantization it turns out that the canonical momentum $p$ has discrete spectrum. One easy way to see this is the following: position and momentum coordinates are related by a Fourier transform. The Fourier transform of a function on a compact space has support only on a discrete space. Now, add a nontrivial smooth bounded potential $V(q)$ to the Hamiltonian describing that system. For generic potentials the quantum dynamics can no longer be solved anymore, that is the 'physical' Hilbert space can no longer be constructed. Nevertheless, the spectrum of the momentum operator is discrete, as is confirmed by numerous experimental results. Thus, from this perspective one would conclude that discreteness of spectra is a kinematical property. In general covariant theories this argument is then further complicated by the interlink between dynamics and constraints.

To summarize, it is still an open question whether the physical spectra of geometrical operators are discrete or not as there are good arguments for both points of view. Much of this confusion is linked to the lack of experience with background independent quantum mechanics, where the principles of standard quantum mechanics will have to be generalized in one way or the other. Unfortunately an answer in the full theory seems out of reach to date, among other things due to mathematical complexity. Symmetry reduced models, such as loop quantum cosmology $[2,9]$, might provide a good test field to study conceptual questions.

\section{Discussion and open questions}

In this article we presented an overview on the topic of observables in gravity. As far as classical gravity is concerned there has been a lot of progress in recent years, at least on the conceptual side. The peculiar case of general relativity, where the gauge group is the diffeomorphism group $\operatorname{Diff}(M)$ and therefore is strongly related to the choice of observer, required the developments of new tools and techniques to cope with mathematical and conceptual difficulties. The method of complete observables encodes in a mathematically precise way the intuitive idea that relations between dynamical quantities are all that matters in a background independent theory. This led to a better understanding of the observable structure of general relativity, which is thought to be an important ingredient for any viable theory of quantum gravity.

However, due to the complicated structure of the gauge group $\operatorname{Diff}(M)$ analytic results for full general relativity coupled to realistic matter fields are to a large extend still missing. The initial question how local gravitational excitations, such as gravitational waves, can be described analytically in a fully gauge- (and thus observer-) invariant way is not answered yet. This is not to say that the problem is poorly understood: there exists a formalism which provides a precise recipe to compute gauge invariant observables for general relativity which is conceptually very well understood. However, calculations are typically so hard that they cannot be solved analytically.

This is a common feature of practically any realistic theory and now one should follow the scheme which has led to success in many cases: stop bothering about formal details, develop approximation methods and extract physical predictions from this formalism.

We described first attempts into this directions in this article, namely the perturbative expansion of complete observables around a fixed spacetime. Within this formalism one can see, among other things, how the observables of linearized gravity (such as gravitational waves) emerge as first order approximations to observables of the full theory. Thus, familiar 'low curvature'physics can be seen to emerge from a fully background independent context. However, as it stands the perturbative formalism is much to complicated to efficiently address questions of true physical interest and needs to be substantially enhanced to be useful for practical calculations. One big unknown, which has a lot of impact on the tractability of the computations, is the choice of clock variables and thus dynamical reference frame.

We discussed a class of model systems with a specific choice of matter fields that leads to a complete de-parameterization when coupled to general relativity. This allows the analytic 
computation of gauge invariant observables for a generally covariant theory and is therefore of outstanding mathematical interest. Unfortunately these matter models seem rather artificial and it is unlikely that they can stand the comparison with phenomenology in gravitational configurations of any kind: the implicit assumption within these models is that there is one single set of clock variables which provides a good reference frame for any possible physical situation.

The latter assumption in a sense contradicts the spirit of general relativity: there might be no preferred reference frame at all and good clock variables have to be chosen case by case, depending on which gravitational configuration one wishes to describe. However, within the framework of complete observables this does a priori not pose any problems. It will be interesting to see whether the analytic developments from the de-parameterized models can be lifted to the general case.

We also reviewed the status of gauge invariant observables in loop quantum gravity. As the physical Hilbert space is so far under poor control the detailed construction of complete quantum observables for gravity has not been possible so far. However, the peculiarities that one has to face when quantizing systems without a global time variable are under investigation and we have discussed a framework that, in the spirit of complete observables, allows to derive different domains of semiunitary evolution from timeless quantum systems. This works as long as there are at least some variables which behave semiclassically and coincides with the expectation that unitary time evolution might become meaningless in the deep quantum regime close to the big bang.

These obstructions can be side-stepped by quantizing the de-parameterizable models in a reduced phase space quantization: as there exists a preferred reference frame and the clock variables are essentially treated as classical all the way down to the deep quantum regime time evolution in that reference frame is implemented unitarily. To date it is unclear how trustworthy the reduced phase space quantization is when physics close to gravitational singularities is concerned, because the quantum fluctuations of the clock variable seem to be artificially suppressed. From this point of view it seems to be important to compare the physical predictions obtained within models based on different reduced phase spaces. Thus, one can check whether they describe the same quantum theory, at least in the semiclassical sector.

For the full theory, where a preferred notion of time in the quantum regime is absent, it will be important to further push the development of approximation tools. We mentioned the mode expansion and the use of effective equations that can convincingly be adapted to timeless quantum theories. We hope that such methods can be generalized to shed further light on the question of quantum observables in full loop quantum gravity.

\section{Acknowledgements}

I want to thank Carlo Rovelli for comments on a previous draft of this article and for helpful suggestions. Discussions with Bianca Dittrich and Wojciech Kaminski are gratefully appreciated. The author is partially supported by the ANR "Programme Blanc" grants LQG-09.

\section{References}

[1] Arnowitt R., Deser S., Misner C.W., The dynamics of general relativity, in Gravitation: an Introduction to Current Research, Wiley, New York, 1962, 227-265.

[2] Ashtekar A., Singh P., Loop quantum cosmology: a status report, Classical Quantum Gravity 28 (2011), 213001, 122 pages, arXiv:1108.0893.

[3] Barbour J., The definition of Mach's principle, Found. Phys. 40 (2010), 1263-1284, arXiv:1007.3368.

[4] Barbour J., The nature of time, arXiv:0903.3489.

[5] Barbour J., Bertotti B., Mach's principle and the structure of dynamical theories, Proc. Roy. Soc. London Ser. A 382 (1982), 295-306. 
[6] Bergmann P.G., "Gauge-invariant" variables in general relativity, Phys. Rev. 124 (1961), 274-278.

[7] Bergmann P.G., Observables in general relativity, Rev. Mod. Phys. 33 (1961), 510-514.

[8] Bergmann P.G., Komar A.B., Poisson brackets between locally defined observables in general relativity, Phys. Rev. Lett. 4 (1960), 432-433.

[9] Bojowald M., Loop quantum cosmology, Living Rev. Relativ. 11 (2008), 4, 131 pages.

[10] Bojowald M., Höhn P.A., Tsobanjan A., An effective approach to the problem of time, Classical Quantum Gravity 28 (2011), 035006, 18 pages, arXiv:1009.5953.

[11] Bojowald M., Höhn P.A., Tsobanjan A., Effective approach to the problem of time: general features and examples, Phys. Rev. D 83 (2011), 125023, 38 pages, arXiv:1011.3040.

[12] Bojowald M., Sandhöfer B., Skirzewski A., Tsobanjan A., Effective constraints for quantum systems, Rev. Math. Phys. 21 (2009), 111-154, arXiv:0804.3365.

[13] Brown J.D., Kuchař K.V., Dust as a standard of space and time in canonical quantum gravity, Phys. Rev. D 51 (1995), 5600-5629, gr-qc/9409001.

[14] Dirac P.A.M., Lectures on quantum mechanics, Belfer Graduate School of Science Monographs Series, Vol. 2, Belfer Graduate School of Science, New York, 1967.

[15] Dirac P.A.M., The theory of gravitation in Hamiltonian form, Proc. Roy. Soc. London. Ser. A 246 (1958), 333-343.

[16] Dittrich B., Partial and complete observables for canonical general relativity, Classical Quantum Gravity 23 (2006), 6155-6184, gr-qc/0507106.

[17] Dittrich B., Partial and complete observables for Hamiltonian constrained systems, Gen. Relativity Gravitation 39 (2007), 1891-1927, gr-qc/0411013.

[18] Dittrich B., Tambornino J., A perturbative approach to Dirac observables and their spacetime algebra, Classical Quantum Gravity 24 (2007), 757-783, gr-qc/0610060.

[19] Dittrich B., Tambornino J., Gauge-invariant perturbations around symmetry-reduced sectors of general relativity: applications to cosmology, Classical Quantum Gravity 24 (2007), 4543-4585, gr-qc/0702093.

[20] Dittrich B., Thiemann T., Are the spectra of geometrical operators in loop quantum gravity really discrete?, J. Math. Phys. 50 (2009), 012503, 11 pages, arXiv:0708.1721.

[21] Domagala M., Giesel K., Kamiński W., Lewandowski J., Gravity quantized: loop quantum gravity with a scalar field, Phys. Rev. D $\mathbf{8 2}$ (2010), 104038, 13 pages, arXiv:1009.2445.

[22] Gambini R., García-Pintos L.P., Pullin J., An axiomatic formulation of the Montevideo interpretation of quantum mechanics, Stud. History Philos. Modern Phys. 42 (2011), 256-263, arXiv:1002.4209.

[23] Gambini R., García-Pintos L.P., Pullin J., Undecidability and the problem of outcomes in quantum measurements, Found. Phys. 40 (2010), 93-115, arXiv:0905.4222.

[24] Gambini R., Porto R.A., Pullin J., Torterolo S., Conditional probabilities with Dirac observables and the problem of time in quantum gravity, Phys. Rev. D $\mathbf{7 9}$ (2009), 041501, 5 pages, arXiv:0809.4235.

[25] Gambini R., Pullin J., Making classical and quantum canonical general relativity computable through a power series expansion in the inverse cosmological constant, Phys. Rev. Lett. 85 (2000), 5272-5275, gr-qc/0008031.

[26] Gambini R., Pullin J., Relational physics with real rods and clocks and the measurement problem of quantum mechanics, Found. Phys. 37 (2007), 1074-1092, quant-ph/0608243.

[27] Gambini R., Pullin J., The large cosmological constant approximation to classical and quantum gravity: model examples, Classical Quantum Gravity 17 (2000), 4515-4539, gr-qc/0008032.

[28] Giddings S.B., Marolf D., Hartle J.B., Observables in effective gravity, Phys. Rev. D 74 (2006), 064018, 20 pages, hep-th/0512200.

[29] Giesel K., Hofmann S., Thiemann T., Winkler O., Manifestly gauge-invariant general relativistic perturbation theory. I. Foundations, Classical Quantum Gravity 27 (2010), 055005, 80 pages, arXiv:0711.0115.

[30] Giesel K., Hofmann S., Thiemann T., Winkler O., Manifestly gauge-invariant general relativistic perturbation theory. II. FRW background and first order, Classical Quantum Gravity 27 (2010), 055006, 52 pages, arXiv:0711.0117.

[31] Giesel K., Tambornino J., Thiemann T., Born-Oppenheimer decomposition for quantum fields on quantum spacetimes, arXiv:0911.5331. 
[32] Giesel K., Tambornino J., Thiemann T., LTB spacetimes in terms of Dirac observables, Classical Quantum Gravity 27 (2010), 105013, 29 pages, arXiv:0906.0569.

[33] Giesel K., Thiemann T., Algebraic quantum gravity (AQG): IV. Reduced phase space quantization of loop quantum gravity, Classical Quantum Gravity 27 (2010), 175009, 29 pages, arXiv:0711.0119.

[34] Guillemin V., Sternberg S., Symplectic techniques in physics, 2nd ed., Cambridge University Press, Cambridge, 1990.

[35] Hellmann F., Partial observables in extended systems, arXiv:0812.0775.

[36] Hellmann F., Mondragon M., Perez A., Rovelli C., Multiple-event probability in general-relativistic quantum mechanics, Phys. Rev. D $\mathbf{7 5}$ (2007), 084033, 13 pages, gr-qc/0610140.

[37] Henneaux M., Teitelboim C., Quantization of gauge systems, Princeton University Press, Princeton, NJ, 1992.

[38] Husain V., Pawlowski T., Dust reference frame in quantum cosmology, arXiv:1108.1147.

[39] Husain V., Pawlowski T., Time and a physical Hamiltonian for quantum gravity, arXiv:1108.1145.

[40] Isham C.J., Canonical quantum gravity and the problem of time, gr-qc/9210011.

[41] Isham C.J., Structural issues in quantum gravity, gr-qc/9510063.

[42] Kamiński W., Lewandowski J., Pawlowski T., Physical time and other conceptual issues of quantum gravity on the example of loop quantum cosmology, Classical Quantum Gravity 26 (2009), 035012, 20 pages, arXiv:0809.2590.

[43] Kamiński W., Lewandowski J., Pawlowski T., Quantum constraints, Dirac observables and evolution: group averaging versus the Schrödinger picture in LQC, Classical Quantum Gravity 26 (2009), 245016, 37 pages, arXiv:0907.4322.

[44] Komar A., Construction of a complete set of independent observables in the general theory of relativity, Phys. Rev. 111 (1958), 1182-1187.

[45] Kuchař K.V., Canonical quantum gravity, gr-qc/9304012.

[46] Kuchař K.V., Time and interpretations of quantum gravity, in Proceedings of the 4th Canadian Conference on General Relativity and Relativistic Astrophysics (Winnipeg, MB, 1991), World Sci. Publ., River Edge, NJ, 1992, 211-314.

[47] Kuchař K.V., Romano J.D., Gravitational constraints that generate a Lie algebra, Phys. Rev. D 51 (1995), 5579-5582, gr-qc/9501005.

[48] Kuchař K.V., Torre C.G., Gaussian reference fluid and interpretation of quantum geometrodynamics, Phys. Rev. D 43 (1991), 419-441.

[49] Kunstatter G., Dirac versus reduced quantization: a geometrical approach, Classical Quantum Gravity 9 (1992), 1469-1485.

[50] Loll R., Noncommutativity of constraining and quantizing: a U(1)-gauge model, Phys. Rev. D 41 (1990), 3785-3791.

[51] Mondragon M., Perez A., Rovelli C., Multiple-event probability in general-relativistic quantum mechanics. II. A discrete model, Phys. Rev. D 76 (2007), 064005, 8 pages, arXiv:0705.0006.

[52] Perez A., Rovelli C., Observables in quantum gravity, gr-qc/0104034.

[53] Plyushchay M.S., Razumov A.V., Dirac versus reduced phase space quantization, in Geometry of Constrained Dynamical Systems (Cambridge, 1994), Cambridge Univ. Press, Cambridge, 1995, 239-250, hep-th/9412137.

[54] Reisenberger M., Rovelli C., Spacetime states and covariant quantum theory, Phys. Rev. D 65 (2002), 125016, 16 pages, gr-qc/0111016.

[55] Rovelli C., A note on the foundation of relativistic mechanics. I. Relativistic observables and relativistic states, gr-qc/0111037.

[56] Rovelli C., A note on the foundation of relativistic mechanics. II. Covariant Hamiltonian general relativity, gr-qc/0202079.

[57] Rovelli C., Comment on "Are the spectra of geometrical operators in Loop Quantum Gravity really discrete?" by B. Dittrich and T. Thiemann, arXiv:0708.2481.

[58] Rovelli C., Dynamics without time for quantum gravity: covariant Hamiltonian formalism and HamiltonJacobi equation on the space $G$, in Decoherence and entropy in complex systems, Lecture Notes in Phys., Vol. 633, Springer, Berlin, 2004, 36-62, gr-qc/0207043. 
[59] Rovelli C., Loop quantum gravity: the first 25 years, Classical Quantum Gravity 28 (2011), 153002, 35 pages, arXiv:1012.4707.

[60] Rovelli C., Quantum gravity, Cambridge Monographs on Mathematical Physics, Cambridge University Press, Cambridge, 2004.

[61] Rovelli C., Quantum mechanics without time: a model, Phys. Rev. D 42 (1990), 2638-2646.

[62] Rovelli C., Partial observables, Phys. Rev. D 65 (2002), 124013, 8 pages, gr-qc/0110035.

[63] Rovelli C., Relational quantum mechanics, Internat. J. Theoret. Phys. 35 (1996), 1637-1678, quant-ph/9609002.

[64] Rovelli C., What is observable in classical and quantum gravity?, Classical Quantum Gravity 8 (1991), 297-316.

[65] Rovelli C., Smolin L., The physical Hamiltonian in nonperturbative quantum gravity, Phys. Rev. Lett. 72 (1994), 446-449, gr-qc/9308002.

[66] Schleich K., Is reduced phase space quantisation equivalent to Dirac quantisation?, Classical Quantum Gravity 7 (1990), 1529-1538.

[67] Speziale S., Background-free propagation in loop quantum gravity, Adv. Sci. Lett. 2 (2009), 280-290, arXiv:0810.1978.

[68] Thiemann T., Modern canonical quantum general relativity, Cambridge Monographs on Mathematical Physics, Cambridge University Press, Cambridge, 2007.

[69] Thiemann T., Reduced phase space quantization and Dirac observables, Classical Quantum Gravity 23 (2006), 1163-1180, gr-qc/0411031.

[70] Thiemann T., Solving the problem of time in general relativity and cosmology with phantoms and $k$-essence, astro-ph/0607380.

[71] Ticciati R., Quantum field theory for mathematicians, Encyclopedia of Mathematics and its Applications, Vol. 72, Cambridge University Press, Cambridge, 1999.

[72] Torre C.G., Gravitational observables and local symmetries, Phys. Rev. D 48 (1993), R2373-R2376, gr-qc/9306030. 